\title{
VARIATIONAL EVOLUTION PROBLEMS AND NONLOCAL GEOMETRIC MOTION
}

\author{
MIKHAIL FELDMAN
}

\begin{abstract}
We consider two variational evolution problems related to MongeKantorovich mass transfer. These problems provide models for collapsing sandpiles and for compression molding. We prove the following connection between these problems and nonlocal geometric curvature motion: The distance functions to surfaces moving according to certain nonlocal geometric laws are solutions of the variational evolution problems. Thus we do the first step of the proof of heuristics developed in earlier works. The main techniques we use are differential equations methods in the Monge-Kantorovich theory.
\end{abstract}

In this paper we study two models involving limits as $p \rightarrow \infty$ of solutions of $p$ Laplacian evolution problems. One is a model of collapsing sandpiles proposed by L. C. Evans, R. F. Gariepy and the author [EFG97]. Another is a model of compression molding proposed by G. Aronsson and L. C. Evans [AE]. In both models the limits were characterized as solutions of variational evolution problems related to MongeKantorovich mass transfer. Solutions that have the form of the distance function to a moving boundary arise naturally in the both models. The equations of the motion of the boundary for both models were derived heuristically in [EFG97] and [AE]. According to the equations the outer normal velocity of the boundary at a point depends on both the local geometry (curvatures) and the nonlocal geometry of the boundary. Thus, the motion of the boundary is a nonlocal geometric motion.

In this paper we prove rigorously the connection between geometric and variational evolution problems. Namely, assuming that a moving surface satisfying the geometric equation is given, we prove that the distance function defines a solution of the corresponding variational evolution problem. The main assumption is that the surface remains convex (or, more generally, semiconvex) during the evolution. For the collapsing sandpiles model we also prove the corresponding result for the solutions that have the form of the maximum of several distance functions (such solutions represent several sand cones interacting in the process of collapse). In the proofs we utilize the connection between the models and the Monge-Kantorovich mass transfer. This allows us to use the differential equations methods in the Monge-Kantorovich theory, which have been developed recently by L. C. Evans and W. Gangbo EGan.

The examples suggest that solutions of the geometric equations derived in [EFG97] can develop singularities, even if the initial data is smooth. Thus we do not assume below that the moving surface is smooth. This however makes the technique more involved.

Research is supported in part by NSF grants DMS-9701755 (MSRI) and DMS-9623276. 
In the forthcoming paper [Fel] we construct convexity preserving viscosity solutions of the geometric equations given convex initial data, thus finishing the proof of the heuristics developed in [EFG97] and [AE].

This paper is organized as following. In Sections 2 - 7 we work on the collapsing sandpiles model. In Section 8 we work on the compression molding model. In Appendix $\mathrm{A}$ we estimate the local Lipschitz constant of the gradient of the distance function of a set.

\section{Notation}

Let $\Omega \in \mathbf{R}^{n}$ be a bounded open set. Let $\Gamma=\partial \Omega$. We will use the following two distance functions of the set $\Omega$. The first, interior distance function, is

$$
d_{\Omega}(x)= \begin{cases}\operatorname{dist}(x, \partial \Omega), & x \in \Omega, \\ 0, & x \in \mathbf{R}^{n} \backslash \Omega .\end{cases}
$$

The second, signed distance function, is

$$
d_{\Omega}^{s}(x)=\left\{\begin{array}{cl}
\operatorname{dist}(x, \partial \Omega), & x \in \Omega, \\
-\operatorname{dist}(x, \partial \Omega), & x \notin \Omega .
\end{array}\right.
$$

Let $x \in R^{n}$. We denote $\mathcal{N}_{\partial \Omega}(x)$ the set of all points of $\partial \Omega$ nearest to $x$, i.e.,

$$
\mathcal{N}_{\partial \Omega}(x)=\left\{y \in \partial \Omega, \quad|x-y|=\inf _{z \in \partial \Omega}|x-z|\right\} .
$$

Note that $\mathcal{N}_{\partial \Omega}(x)$ may have more then one point.

Let $y \in \partial \Omega$. Then $\partial \Omega$ is differentiable at $y$ if there exists $\delta>0$ such that in a suitable coordinate system $\left(x_{1}, \ldots, x_{n}\right)$ in $\mathbf{R}^{n}$ we have

$$
y=0, \quad \Omega \cap B_{\delta}(0)=\left\{x_{n}>\Psi\left(x^{\prime}\right)\right\} \cap B_{\delta}(0) \quad \text { where } \quad \Psi\left(x^{\prime}\right)=o\left(\left|x^{\prime}\right|\right) .
$$

Here $x^{\prime}=\left(x_{1}, \ldots, x_{n-1}\right)$, and $B_{\delta}(0)=B_{\delta}^{n}(0)$ is the ball in $\mathbf{R}^{n}$ with radius $\delta$ and center at $0 . \partial \Omega$ is twice differentiable at $y$ if in a suitable coordinate system in $\mathbf{R}^{n-1}$ the function $\Psi$ has the expression

$$
\Psi\left(x^{\prime}\right)=\sum_{i=1}^{n-1} \kappa_{i} x_{i}^{2}+o\left(\left|x^{\prime}\right|^{2}\right) .
$$

The numbers $\kappa_{1}, \ldots, \kappa_{n-1}$ are the principal curvatures of $\partial \Omega$ at $y$.

Let $\left\{\Omega_{t}\right\}, t \in \mathbf{R}_{+}^{1}$, be a family of open bounded sets in $\mathbf{R}^{n}$. We call a family of sets $\left\{\Omega_{t}\right\}$ Lipschitz (with respect to $t$ ) if there exists a constant $M>0$ such that for any $t_{1}, t_{2}$ the set $\left(\Omega_{t_{1}} \backslash \Omega_{t_{2}}\right) \cup\left(\Omega_{t_{2}} \backslash \Omega_{t_{1}}\right)$ is subset of both $M\left|t_{1}-t_{2}\right|$ neighborhood (in $\mathbf{R}^{n}$ ) of $\partial \Omega_{t_{1}}$ and $M\left|t_{1}-t_{2}\right|$ neighborhood of $\partial \Omega_{t_{2}}$.

Let

$$
\begin{aligned}
& E=\cup_{t}\left(\Omega_{t} \times\{t\}\right) \subset \mathbf{R}^{n} \times \mathbf{R}_{+}^{1}, \\
& \Gamma=\cup_{t}\left(\partial \Omega_{t} \times\{t\}\right) \subset \mathbf{R}^{n} \times \mathbf{R}_{+}^{1}, \\
& \Gamma_{t}=\partial \Omega_{t} \subset \mathbf{R}^{n} .
\end{aligned}
$$


Let $(y, t) \in \mathbf{R}^{n} \times \mathbf{R}_{+}^{1}$ be such that $y \in \Gamma_{t}$ and the function $(x, \tau) \rightarrow d_{\Omega_{\tau}}^{s}(x)$ is differentiable at $(y, t)$. We define the outer normal velocity of $\Gamma_{t}$ at $y$ as

$$
V(y)=\frac{\partial}{\partial t} d_{\Omega_{t}}^{s}(y)
$$

Let $\left(y, t_{0}\right) \in \Gamma$. The surface $\Gamma$ is $(2,1)$ differentiable at $\left(y, t_{0}\right)$ if in a suitable coordinate system $\left(x_{1}, \ldots, x_{n}\right)$ in $\mathbf{R}^{n}$ we have $y=0, E \cap B_{\delta}^{n+1}(0)=\left\{x_{n}>\Psi\left(x^{\prime}, t\right)\right\} \cap$ $B_{\delta}^{n+1}(0)$ for small $\delta>0$ and

$$
\Psi\left(x^{\prime}, t\right)=v\left(t-t_{0}\right)+\sum_{i=1}^{n-1} \kappa_{i} x_{i}^{2}+o\left(\left|x^{\prime}-y^{\prime}\right|^{2}+\left|t-t_{0}\right|\right) .
$$

$\mathcal{L}^{n}$ is $n$-dimensional Lebesgue measure.

$\mathcal{H}^{n}$ is $n$-dimensional Hausdorff measure.

\section{The COLlapsing SANDPILES MODEL}

In the paper EFG97 the $p$-Laplacian evolution problems

$$
\begin{cases}\partial_{t} u_{p}-\operatorname{div}\left(\left|D u_{p}\right|^{p-2} D u_{p}\right)=0, & \text { in } \mathbf{R}^{n} \times(0, \infty), \\ u_{p}=g, & \text { on } \mathbf{R}^{n} \times\{0\},\end{cases}
$$

are considered in the "infinitely fast/slow diffusion limit" $p \rightarrow \infty$. Du $u_{p}$ denotes the gradient of $u_{p}$ with respect to the spatial variables $x_{1}, \ldots, x_{n}$. The operator

$$
\Delta_{p}(u)=\operatorname{div}\left(\left|D u_{p}\right|^{p-2} D u_{p}\right)
$$

is $p$-Laplacian. The initial data $g$ is nonnegative, Lipschitz continuous function with compact support. We assume that

$$
\operatorname{Lip}[g]=L>1
$$

where $\operatorname{Lip}[g]$ is the Lipschitz constant of $g$.

The initial data $g$ satisfying (8) is unstable in the following sense. It is shown in EFG97 that $u_{p} \rightarrow u$ uniformly on $\mathbf{R}^{n} \times(0, \infty)$, and that

$$
|D u| \leq 1 \quad \text { a.e. in } \mathbf{R}^{n} \times(0, \infty) .
$$

This limit $u$ does not depend on $t$ for $t>0$, i.e., $u=u(x)$. By (8) and (9) we get $u \neq g$. Thus the limit solution is discontinuous at $t=0$. The transformation $g \rightarrow u$ takes place at $t=0$.

The described problem can be interpreted as a crude model of a collapsing sandpile. The function $u(x, t)$ is the height of the pile at the location $x$, time $t$. The main physical assumption is that a sandpile is stable if its slope does not exceed 1, i.e., if $\operatorname{Lip}[u(\cdot, t)] \leq 1$. The condition (8) implies that the initial profile $g$ is unstable. According to the model above, the initially unstable sandpile collapses instantaneously to a stable one, with the height function $u$.

The transformation $g \rightarrow u$ was studied in [EFG97 by relating it to the evolution problem governed by Monge-Kantorovich mass transfer. Introduce the convex 
functional $I_{\infty}: L^{2}\left(\mathbf{R}^{n}\right) \rightarrow \mathbf{R}^{1} \cup\{\infty\}$ defined by

$$
I_{\infty}[v]= \begin{cases}0 & \text { if } v \in L^{2}\left(\mathbf{R}^{n}\right), \quad|D v| \leq 1 \text { a.e. } \\ +\infty & \text { otherwise. }\end{cases}
$$

Then $u(x)=w(x, 1)$, where $w$ is the unique solution of the evolution problem

$$
\begin{cases}\frac{w}{t}-\partial_{t} w \in \partial I_{\infty}[w], & \text { for a.e. } t \in\left[\frac{1}{L}, 1\right], \\ w=\frac{g}{L}=\hat{g}, & \text { at } t=\frac{1}{L},\end{cases}
$$

with $L=\operatorname{Lip}[g]>1$. Note that $\operatorname{Lip}[\hat{g}]=1$, and thus the evolution problem (10) is well-posed. The connection between this evolution problem and Monge-Kantorovich mass transfer is explained in EFG97.

In the static case the limits as $p \rightarrow \infty$ of the boundary value problems for $p$ Laplacian type equations were considered in the papers BDM89, Jan93 and references therein. In many cases the limits have the form of the distance function to the boundary of the domain.

A limit evolution problem that represents a stable case of the problem (7) was considered in the paper [AEW96] as a model of a growing stable sandpile. Let $u(x, t)$ be, as before, the height of the pile, and let $f(x, t) \geq 0$ be the sand source function which describes the rate at which sand is coming at location $x$ at time $t$. The dynamics is defined as following. Consider the nonhomogeneous evolution problem

$$
\begin{cases}\partial_{t} u_{p}-\operatorname{div}\left(\left|D u_{p}\right|^{p-2} D u_{p}\right)=f, & \text { in } \mathbf{R}^{n} \times(0, \infty), \\ u_{p}=0, & \text { on } \mathbf{R}^{n} \times\{0\},\end{cases}
$$

and let $p \rightarrow \infty$. Then for any $T>0$

$$
u_{p} \rightarrow u \quad \text { uniformly on } \mathbf{R}^{n} \times[0, T]
$$

and this limit $u$ satisfies the equation

$$
f-\partial_{t} u \in \partial I_{\infty}[u] \text {, for a.e. } t \geq 0
$$

with zero initial condition. In particular the case was considered when sand sources are concentrated in $m$ points, i.e., if

$$
f(x, t)=\sum_{k=1}^{m} f_{k}(t) \delta_{d_{k}}(x),
$$

where $\delta_{d_{k}}(\cdot)$ is the Dirac mass at the point $d_{k} \in \mathbf{R}^{n}$. The solution has the explicit form

$$
u=\max \left(0, z_{1}(t)-\left|x-d_{1}\right|, \ldots, z_{m}(t)-\left|x-d_{m}\right|\right),
$$

where the height functions $\left\{z_{k}(t)\right\}_{k=1}^{m}$ satisfy a system of ODE. Thus the solution has the form of growing and interacting cones centered at the points $\left\{d_{k}\right\}_{k=1}^{m}$.

By analogy with the static problems and the model of growing sandpiles [AEW96], one can expect to find solutions of (10) that have the form of distance function to the moving boundary or superposition of such, i.e., growing and interacting cones. However, unlike the situation considered in [AEW96], interacting spherical cones do not maintain their shape under the evolution defined by (10). A more suitable class 
of solutions is the following class of more general "cones". A single cone solution has the form

$$
w(x, t)=d_{\Omega_{t}}(x)
$$

where $\left\{\Omega_{t}\right\}$ is a suitable expanding family of sets. A multiple interacting cones solution has the form (for simplicity we consider the case of two cones)

$$
w(x, t)=\max \left(d_{\Omega_{t}^{1}}(x), d_{\Omega_{t}^{2}}(x)\right),
$$

where $\left\{\Omega_{t}^{1}\right\},\left\{\Omega_{t}^{2}\right\}$ are suitable expanding families of sets. Thus in order to find such solutions one has to find the appropriate families of sets.

The following equations of motion of the boundaries of the sets in (13) and (14) are derived heuristically in [EFG97]. Assume that $\Gamma$ defined by (5) is a smooth surface in $\mathbf{R}^{n} \times \mathbf{R}^{1}$. Let $y \in \Gamma_{t}$ and let $\kappa_{1}, \ldots, \kappa_{n-1}$ be the principal curvatures of $\Gamma_{t}$ at $y$. Denote $\kappa=\left(\kappa_{1}, \ldots, \kappa_{n-1}\right)$. Let $\gamma(y)=\gamma_{\Omega_{t}}(y)$ be the radius of the largest ball touching $\Gamma_{t}$ at $y$ from within $\Omega_{t}$. Let $V(y)$ be the outer normal velocity of $\Gamma_{t}$ at the point $y$ defined by (6). Then in the case of a single cone solution (13) the equation for the surface $\Gamma_{t}$ is:

$$
V(y)=\frac{1}{t} F(\kappa(y), \gamma(y)) \text { for } y \in \Gamma_{t},
$$

where

$$
F(\kappa, \gamma)=\frac{\int_{0}^{\gamma} s \prod_{i=1}^{n-1}\left(1-\kappa_{i} s\right) d s}{\int_{0}^{\gamma} \prod_{i=1}^{n-1}\left(1-\kappa_{i} s\right) d s} .
$$

To write equations for the motion of the surfaces $\Gamma_{t}^{k}=\partial \Omega_{t}^{k}, k=1,2$ in the case of the interacting cones solution (14), we extend the function $\gamma(\cdot)=\gamma_{\Omega}(\cdot)$ of a set $\Omega \in \mathbf{R}^{n}$ to a function $\mathbf{R}^{n} \rightarrow \mathbf{R}^{1}$ by setting $\gamma \equiv 0$ on $\mathbf{R}^{n} \backslash \bar{\Omega}$, and $\gamma(x)=\gamma(y)$ for $x \in \Omega$ such that $y \in \mathcal{N}_{\partial \Omega}(x)$. Then the equations are:

$$
V(y) \quad=\frac{1}{t} F\left(\kappa(y), \gamma_{\Omega_{t}^{k}}(y), \gamma_{\Omega_{t}^{1} \cap \Omega_{t}^{2}}(y)\right) \text { for } y \in \Gamma_{t}^{k}, k=1,2,
$$

where

$$
F(\kappa, \gamma, \delta)=\frac{\int_{\delta}^{\gamma} s \prod_{i=1}^{n-1}\left(1-\kappa_{i} s\right) d s}{\int_{\delta}^{\gamma} \prod_{i=1}^{n-1}\left(1-\kappa_{i} s\right) d s} .
$$

The equations (15) and (16) have the form of nonlocal geometric curvature motion. Geometric nonlocality is caused by the functions $\gamma(\cdot)$.

In this paper we prove that if the moving surfaces satisfy geometric equations (15) or (16) and some regularity conditions, then the functions (13) or (14) respectively are solutions of the evolution problem (10). Namely, we prove the following.

Theorem 1. Let $\left\{\Omega_{t}\right\}$ be a locally Lipschitz continuous family of open convex bounded sets, where $t \in[a, b], b>a>0$. Suppose that the equation (15) is satisfied at every point of $(2,1)$ differentiability of the surface $\Gamma$ defined by (5). Then the function (13) is a solution of the evolution equation

$$
\frac{w}{t}-\partial_{t} w \in \partial I_{\infty}[w] \quad \text { for a.e. } t \in[a, b] .
$$


Theorem 2. Let $\left\{\Omega_{t}^{1}\right\},\left\{\Omega_{t}^{2}\right\}$ be two Lipschitz continuous families of open bounded convex sets, where $t \in[a, b], b>a>0$. Suppose that the equation (10) is satisfied at every point of $(2,1)$ differentiability of the surfaces $\Gamma^{k}=\cup_{t}\left(\Gamma_{t}^{k} \times\{t\}\right), k=1,2$. Then the function (14) is a solution of the evolution equation (17).

The convexity condition in Theorems 1, 2 can be relaxed to the following "semiconvexity" condition.

Condition 3. (lower curvature bound condition with radius $r$ ). An open set $\Omega \subset \mathbf{R}^{n}$ satisfies this condition if $\gamma_{\mathbf{R}^{n} \backslash \bar{\Omega}}(x) \geq 2 r$ for any $x \in \mathbf{R}^{n} \backslash \bar{\Omega}$.

It is easy to check that if a set is either convex or has $C^{1,1}$ boundary, then the set satisfies the Condition 3 .

Remark 4. Let the set $\Omega$ satisfy Condition 3 . Let $\partial \Omega$ be twice differentiable at a point $y$, i.e., (3) and (4) are satisfied in an appropriate coordinate system on $\mathbf{R}^{n}$. Then the principal curvatures of $\partial \Omega$ at $y$ satisfy

$$
\kappa_{i} \geq-\frac{1}{2 r} \quad \text { for } i=1, \ldots, n-1 .
$$

This follows from the fact that the graph of the function $\Psi$ defined by (3) and (4) lies above the ball $B_{2 r}^{n}(0,-2 r)$ by Condition 8 .

The following two theorems state that convexity can be replaced by the weaker Condition 3 in Theorems 11 and 2 .

Theorem 5. Let $\left\{\Omega_{t}\right\}$ be a locally Lipschitz continuous family of open bounded sets, where $t \in[a, b], b>a>0$. Let for every $t \in[a, b]$ the set $\Omega_{t}$ satisfy Condition 3 with radius $r_{0}>0$. Suppose that the equation (15) is satisfied at every point of $(2,1)$ differentiability of the surface $\Gamma$ defined by (5). Then the function (13) is a solution of the evolution equation (17).

Theorem 6. Let $\left\{\Omega_{t}^{1}\right\},\left\{\Omega_{t}^{2}\right\}$ be two Lipschitz continuous families of open bounded sets, where $t \in[a, b], b>a>0$. Let for every $t \in[a, b]$ the sets $\Omega_{t}^{1}$, $\Omega_{t}^{2}$ satisfy Condition 3 with radius $r_{0}>0$. Suppose that the equation (10) is satisfied at every point of $(2,1)$ differentiability of the surfaces $\Gamma^{k}=\cup_{t}\left(\Gamma_{t}^{k} \times\{t\}\right), k=1,2$. Then the function (14) is a solution of the evolution equation (17).

Theorems 1 and 2 follow from Theorems 5 and 6 since convex sets satisfy Condition 3 with any radius.

The proof of Theorems 5 and 6 utilizes the following relation of the equation (17) and the Monge-Kantorovich mass transfer problem. Fix $t$. Equation (17) implies that that the function $w(\cdot, t)$ is the Monge's potential for the optimal transfer of the measure with density $\frac{w}{t}(\cdot, t)$ into the measure with density $\partial_{t} w(\cdot, t)$. Then, according to [EGan], there exists a measurable function $a(x)$, the mass transport density, that satisfies the following properties:

$$
\begin{aligned}
a \geq 0, & \operatorname{supp}(a) \subset\{x|| D w \mid=1\}, \\
-\operatorname{div}(a D w)= & \frac{w}{t}-\partial_{t} w,
\end{aligned}
$$


where the last equation is understood in the weak sense and $t$ is fixed. Conversely, if there exists a function $a(x)$ satisfying (18), then the function $w$ is a solution of (17) for the given $t$, see Section 6 below.

The idea of the proof of Theorem 5 is as follows. Let a family of sets $\left\{\Omega_{t}\right\}$ satisfy (13). An open bounded set can be represented as the union of distance rays, i.e., maximal intervals that start at the boundary, on which distance to the boundary is the linear function with slope 1 . The Condition 3 implies that the collection of distance rays possesses "nice" measure-theoretic properties. Fix $t$ and consider a distance ray $R_{y}$ of $\Omega_{t}$ starting at a smooth point $y \in \partial \Omega_{t}$. Introduce the coordinate $s$ on $R_{y}$, the distance to the boundary. The equation (18) can be formally rewritten as:

$$
D a \cdot D w+a \Delta w-\frac{w}{t}-\partial_{t} w=0 .
$$

Since $w(\cdot, t)=\operatorname{dist}\left(\cdot, \Gamma_{t}\right)$, we have on $R_{y}$

$$
D a \cdot D w=\frac{d a}{d s}, \quad w=s, \quad-\Delta w=\sum_{i=1}^{n-1} \frac{\kappa_{i}}{1-\kappa_{i} s}, \quad \partial_{t} w=V(y),
$$

where $\kappa_{1}, \ldots, \kappa_{n-1}$ are the principal curvatures of $\Gamma_{t}$ at $y$, and $V(y)$ is the outer normal velocity of $\Gamma_{t}$ at $y$. Thus the equation (19) can be formally rewritten on the ray $R_{y}$ as the following ODE

$$
a^{\prime}(s)-a(s) \sum_{i=1}^{n-1} \frac{\kappa_{i}}{1-\kappa_{i} s}-\frac{s}{t}+V(y)=0,
$$

This ODE has a solution $a(s)$ with zero boundary conditions at both ends of the ray $R_{y}$ if $V(y)$ satisfies (15). Define $a(x, t)$ by ODE (20) with zero boundary conditions on each distance ray that starts at a smooth point of the boundary. We prove that $a(\cdot, t)$ is a measurable function that satisfies (18). In order to do this we examine the properties of distance function and distance rays, and use a nonsmooth (Lipschitz) change of coordinates on the set $\Omega_{t}$ with $n-1$ coordinates along the boundary and the $n$-th coordinate along distance rays.

We prove Theorems 5 and 6 in Sections 3 - 7. In Section 3 we examine properties of sets satisfying Condition 3, and in particular we describe the change of coordinates mentioned above. In Section 1 we define the mass transport density function and examine some properties of this function. In Section 5 we prove that the mass balance equation (15) is satisfied. In Section 6 we conclude the proof of Theorem 5 . Finally, in Section 7 we sketch the proof of Theorem 6 .

\section{Properties of Distance Function And Ridge Sets of the Sets SATISFYING THE LOWER CURVATURE BOUND CONDITION}

Let $\Omega$ be an open set. When there is no possible confusion, we write $d(x)$ for $d_{\Omega}(x)$ defined by (1).

Let $x \in \bar{\Omega}$. Denote by $R_{x}$ the longest line segment through $x$ in $\bar{\Omega}$ along which $d_{\Omega}(\cdot)$ is a linear function with slope 1 . We call $R_{x}$ the distance ray of $x$. Note that a point $x$ can have more than one distance ray, in such case denote any of them by $R_{x}$. 
If $x \in \Omega$ then all such rays $R_{x}$ have equal length. If $x \in \partial \Omega$ then rays $R_{x}$ in the case of general open set $\Omega$ can have different length. However if $\Omega$ satisfies Condition 3 then there exists at most one $R_{x}$ for $x \in \partial \Omega$, see Proposition 9 below.

For any $x \in \Omega$ one endpoint of $R_{x}$ lies on $\partial \Omega$ and belongs to the set $\mathcal{N}_{\partial \Omega}(x)$. Call this endpoint the lower end of $R_{x}$. Call another endpoint of $R_{x}$ the upper end of $R_{x}$ (the names "lower" and "upper" correspond to the positions of the endpoints of $R_{x}$ on the graph of $\left.y=d_{\Omega}(x)\right)$. Let $y, z$ be upper and lower ends of the ray $R$, and let $x=\lambda y+(1-\lambda) z$ where $\lambda \in(0,1)$. Then we say that the point $x$ lies in the relative interior of the ray $R$.

Remark 7. If $x \in \Omega$ lies in the relative interior of a distance ray $R_{x}$, then the ray $R_{x}$ is the unique distance ray that contains $x$, and the set $\mathcal{N}_{\partial \Omega}(x)$ consists of one point, see EH87.

Remark 8. It follows from Remark 0 that sets satisfying Condition 0 are sets with positive reach defined by $H$. Federer [Fed59]. More precisely, if a set $\Omega$ satisfies Condition 3 with radius $r$ then $\operatorname{reach}(\Omega)=2 r$.

The function $\gamma_{\Omega}(\cdot)$ in the equations $(\sqrt{15})$, (16) is called ridge function and can be expressed as following:

$$
\gamma_{\Omega}(x)= \begin{cases}0 & x \in \mathbf{R}^{n} \backslash \bar{\Omega} \\ \left|R_{x}\right| & x \in \Omega \\ \sup \left|R_{x}\right| & x \in \partial \Omega\end{cases}
$$

The function $\gamma_{\Omega}(\cdot)$ is uppersemicontinuous in $\mathbf{R}^{n}$, cf. [EH87], Proposition 3.2.

Define the set

$$
\mathcal{R}=\left\{x \in \bar{\Omega} \quad \mid d_{\Omega}(x)=\gamma_{\Omega}(x)\right\} .
$$

The set $\mathcal{R}$ is called ridge set of $\Omega$. Note that if $x \in \mathcal{R}$ then either $x$ is the upper end of some distance ray of $\Omega$, or otherwise $x$ lies at $\partial \Omega$ and there are no points $y \in \Omega$ such that $x \in \mathcal{N}_{\partial \Omega}(y)$.

Proposition 9. Let $\Omega \subset \mathbf{R}^{n}$ be a bounded open set satisfying Condition 3 with radius $r>0$. Let $\Omega_{r}$ be the $r$-neighborhood of $\Omega$. Then

(a) $\gamma_{\mathbf{R}^{n} \backslash \bar{\Omega}_{r}}(x) \geq r$ for all $x \in \mathbf{R}^{n} \backslash \Omega_{r}$, i.e., the set $\Omega_{r}$ satisfies Condition $\Omega$ with radius $\frac{r}{2}$.

(b) For any $y_{r} \in \partial \Omega_{r}$ there exists a unique $y \in \partial \Omega$ such that $\left|y_{r}-y\right|=r$, and $\left|y_{r}-x\right|>r$ for all $x \in \bar{\Omega} \backslash\{y\}$. For any $y \in \partial \Omega$ there exists $y_{r} \in \partial \Omega_{r}$ such that $\left|y-y_{r}\right|=r$. If $\partial \Omega$ is differentiable at $y$ then $y-y_{r}$ is orthogonal to $\partial \Omega$ at $y$. If there exists a ball inside $\Omega$ touching $\partial \Omega$ at $y$, then $\partial \Omega$ is differentiable at $y$. In particular, $\partial \Omega_{r}$ is everywhere differentiable.

(c) For any $x \in \Omega$ we have

$$
\operatorname{dist}\left(x, \partial \Omega_{r}\right)=\operatorname{dist}(x, \partial \Omega)+r .
$$

If $x \in \Omega_{r} \backslash \bar{\Omega}$ then $\operatorname{dist}\left(x, \partial \Omega_{r}\right)<r$ and $x \notin \mathcal{R}_{r}$, where $\mathcal{R}_{r}$ is ridge set of $\Omega_{r}$.

(d) Sets $\Omega$ and $\Omega_{r}$ have the same ridge set, i.e., $\mathcal{R}=\mathcal{R}_{r}$. 
Proof. (a) Let $x \in \mathbf{R}^{n} \backslash \bar{\Omega}_{r}$. Then $\operatorname{dist}(x, \Omega)=R>r$. Let $y \in \partial \Omega$ be a point such that $|x-y|=R$. Let $y_{r}$ be such point of the interval connecting $x$ and $y$ that $\left|y-y_{r}\right|=r$. Then $y_{r} \in \bar{\Omega}_{r}$.

Consider first the case $R \geq 2 r$. Then $\operatorname{dist}\left(x, \Omega_{r}\right) \geq r$. Since if this is not true then there exists such point $z_{1} \in \bar{\Omega}_{r}$ that $\left|x-z_{1}\right|<r$, and there exists $z_{2} \in \bar{\Omega}$ such that $\left|z_{1}-z_{2}\right| \leq r$. Now we get $R \leq\left|x-z_{2}\right| \leq\left|x-z_{1}\right|+\left|z_{1}-z_{2}\right|<2 r$, a contradiction. So $\gamma_{\mathbf{R}^{n} \backslash \bar{\Omega}_{r}}(x) \geq \operatorname{dist}\left(x, \Omega_{r}\right) \geq r$.

Consider now the case $r<R<2 r$. Then by Condition 3 we have $\gamma_{\mathbf{R}^{n} \backslash \bar{\Omega}}(x) \geq 2 r$ and so $R=\operatorname{dist}(x, \Omega)<\gamma_{\mathbf{R}^{n} \backslash \bar{\Omega}}(x)$. Thus $y$ is the unique point on $\partial \Omega$ such that $|x-y|=\operatorname{dist}(x, \Omega)$. Let $z=y+2 r \frac{x-y}{|x-y|}$. Then from the condition 3 and [EH87, Lemma 3.4, it follows that for any $\tilde{x}$ of the form $t x+(1-t) z$ where $0<t<1$ the point $y$ is the unique point on $\partial \Omega$ such that $\operatorname{dist}(\tilde{x}, \partial \Omega)=|\tilde{x}-y|$. Then for each such point $\tilde{x}$, the point $y_{r}$ is the unique point on $\partial \Omega_{r} \operatorname{such}$ that $\operatorname{dist}\left(\tilde{x}, \partial \Omega_{r}\right)=\left|\tilde{x}-y_{r}\right|$. For suppose on the contrary that there exists $z_{1} \in \partial \Omega_{r}$ such that $z_{1} \neq y_{r}$ and $\left|\tilde{x}-z_{1}\right| \leq\left|\tilde{x}-y_{r}\right|$. Then there exists $z_{2} \in \partial \Omega$ such that $\left|z_{1}-z_{2}\right| \leq r$. Consider first the case $z_{2} \neq y$. Then

$$
|\tilde{x}-y|=\left|\tilde{x}-y_{r}\right|+\left|y_{r}-y\right|=\left|\tilde{x}-y_{r}\right|+r \geq\left|\tilde{x}-z_{1}\right|+\left|z_{1}-z_{2}\right| \geq\left|\tilde{x}-z_{2}\right|,
$$

a contradiction with the fact that $y$ is the unique nearest to $\tilde{x}$ point on $\partial \Omega$. Consider now the case $z_{2}=y$. Since the ball of radius $R$ and center $\tilde{x}$ lies outside $\Omega$ and $\left|z_{1}-y\right| \leq r$, it follows that $z_{1}$ does not lie on the interval connecting $\tilde{x}$ and $y$, and so

$$
|\tilde{x}-y|<\left|\tilde{x}-z_{1}\right|+\left|z_{1}-y\right| \leq\left|\tilde{x}-y_{r}\right|+r=|\tilde{x}-y|
$$

a contradiction. So, $y_{r}$ is the unique nearest to $\tilde{x}$ point on $\partial \Omega_{r}$ for any $\tilde{x}$ on the interval connecting $z$ and $y_{r}$, so $\gamma_{\mathbf{R}^{n} \backslash \bar{\Omega}_{r}}(x) \geq\left|z-y_{r}\right|=r$.

(b) Let $y_{r} \in \partial \Omega_{r}$. Then $\operatorname{dist}\left(y_{r}, \Omega\right)=r$. Thus there exists $y \in \partial \Omega$ such that $\left|y-y_{r}\right|=r$. By Condition $3, \gamma_{\mathbf{R}^{n} \backslash \bar{\Omega}}\left(y_{r}\right) \geq 2 r>\operatorname{dist}\left(y_{r}, \partial \Omega\right)$, and thus by [EH87, Lemma 3.4, $y$ is the unique point of $\partial \Omega$ nearest to $y_{r}$.

Let $y \in \partial \Omega$. Let $y_{i} \in \mathbf{R}^{n} \backslash \bar{\Omega}$ for $i=1,2, \ldots$, and let $y_{i} \rightarrow y$. By condition 3 , $\gamma_{\mathbf{R}^{n} \backslash \bar{\Omega}}\left(y_{i}\right) \geq 2 r$, and by uppersemicontinuity of $\gamma_{\mathbf{R}^{n} \backslash \bar{\Omega}}(\cdot)$ we get $\gamma_{\mathbf{R}^{n} \backslash \bar{\Omega}}(y) \geq 2 r$. Thus we have shown that every point of $\partial \Omega$ has an exterior tangent ball of the radius $2 r$. So if a point $y$ of $\partial \Omega$ has an interior tangent ball, then $\partial \Omega$ is differentiable at $y$.

Let $y \in \partial \Omega$. Then, as we have shown, $\gamma_{\mathbf{R}^{n} \backslash \bar{\Omega}}(y) \geq 2 r$. Thus there exists a distance ray $R_{y}$ of the set $\mathbf{R}^{n} \backslash \bar{\Omega}$ starting at $y$ such that the length of $R_{y}$ is at least $2 r$. Let $e$ be the unit vector in the direction of $R_{y}$, let $y_{r}=y+r e$. Then $\mathcal{N}_{\partial \Omega}\left(y_{r}\right)=\{y\}$ by Remark 7. Since $\left|y-y_{r}\right|=r$ we get $y_{r} \in \partial \Omega_{r}$.

We have shown that the interval connecting $y$ and $y_{r}$ lies on the distance ray of the set $\mathbf{R}^{n} \backslash \bar{\Omega}$ starting at $y$, and on distance ray of the set $\Omega_{r}$ starting at $y_{r}$. It follows that the vector $y-y_{r}$ is orthogonal to $\partial \Omega_{r}$ at $y_{r}$ and also to $\partial \Omega$ at $y$ if $\partial \Omega$ is differentiable at $y$. 
(c) Let $x \in \Omega^{0}, y \in \partial \Omega$, and $|x-y|=\operatorname{dist}(x, \partial \Omega)$. Then, by (b),

$$
y_{r}:=y+\frac{r}{|y-x|}(y-x) \in \partial \Omega_{r} .
$$

Clearly,

$$
\left|x-y_{r}\right|=|x-y|+r=d_{\Omega}(x)+r .
$$

Assume that there exists $z_{r} \in \partial \Omega_{r}$ such that

$$
\left|x-z_{r}\right|<d_{\Omega}(x)+r .
$$

Let $z$ be the point of intersection of $\partial \Omega$ with the interval connecting $z_{r}$ and $x$ (such point exists since $\left.x \in \Omega, z_{r} \notin \bar{\Omega}\right)$. Then $\left|z-z_{r}\right| \geq r$ and so

$$
|x-z|<d_{\Omega}(x) \text {. }
$$

This contradicts the fact that $z \in \partial \Omega$. Thus $y_{r} \in \mathcal{N}_{\partial \Omega_{r}}(x)$ and thus (22) is proved.

It remains to prove the last assertion of the statement (c).

Let $x \in \Omega_{r} \backslash \bar{\Omega}$. Let $y_{r} \in \mathcal{N}_{\partial \Omega_{r}}(x)$. Let $R_{y_{r}}$ be the ray orthogonal to $\partial \Omega_{r}$ at $y_{r}$ and let $y=R_{y_{r}} \cap \partial \Omega$. Then by (b) we have the following: $x \in R_{y_{r}}, x$ lies on the interval connecting $y$ and $y_{r}$, and $\left|y-y_{r}\right|=r=\operatorname{dist}\left(y, \partial \Omega_{r}\right)$. Then it follows from [EH87, Lemma 3.4 that $x \notin \mathcal{R}_{r}$. Also, $\operatorname{dist}\left(x, \partial \Omega_{r}\right)=\left|x-y_{r}\right|<\left|y-y_{r}\right|=r$.

(d) We show first that $\mathcal{R} \subset \mathcal{R}_{r}$. If $x \in \Omega$ and $B_{\rho}(x) \subset \Omega$, then $B_{\rho+r}(x) \subset \bar{\Omega}_{r}$. Let $x \in \mathcal{R} \backslash \partial \Omega$. Let $y \in \mathcal{N}_{\partial \Omega}(x)$ and let $y_{r}=y+r \frac{y-x}{|y-x|}$. Then by (b),(c) we see that $y_{r} \in \partial \Omega_{r}$, and $\left|x-y_{r}\right|=\operatorname{dist}(x, \partial \Omega)+r=\operatorname{dist}\left(x, \partial \Omega_{r}\right)$. Let $\tilde{x}=y+(1+\varepsilon)(x-y)$ where $\varepsilon>0$ is small enough so that $\tilde{x} \in \Omega$. Since $x \in \mathcal{R}$, it follows that $\operatorname{dist}(\tilde{x}, \partial \Omega)<$ $|\tilde{x}-y|=(1+\varepsilon) \operatorname{dist}(x, \partial \Omega)$. Let $\tilde{y} \in \partial \Omega$ be such point that $\operatorname{dist}(\tilde{x}, \partial \Omega)=|\tilde{x}-\tilde{y}|$, let $\tilde{y}_{r}=\tilde{y}+r \frac{\tilde{y}-\tilde{x}}{|\tilde{y}-\tilde{x}|}$, then $\tilde{y}_{r} \in \partial \Omega_{r}$ and $\left|\tilde{x}-\tilde{y}_{r}\right|=\operatorname{dist}\left(\tilde{x}, \partial \Omega_{r}\right)$. Then

$$
\operatorname{dist}\left(\tilde{x}, \partial \Omega_{r}\right)=|\tilde{y}-\tilde{x}|+r<(1+\varepsilon) \operatorname{dist}(x, \partial \Omega)+r=\left|\tilde{x}-y_{r}\right|,
$$

so $\gamma_{\Omega_{r}}(x)=\left|x-y_{r}\right|=d_{\Omega_{r}}(x)$. Thus $x \in \mathcal{R}_{r}$.

Let $x \in \mathcal{R} \cap \partial \Omega$, i.e., there does not exist a ball inside $\Omega$ that touches $\partial \Omega$ at $x$. Let $B_{r}\left(y_{r}\right)$ be a ball of radius $r$ and center at such point $y_{r}$ that $\bar{B}_{r}\left(y_{r}\right) \cap \bar{\Omega}=\{x\}$, such ball exists by Condition 3. Then $\operatorname{dist}\left(y_{r}, \partial \Omega\right)=r$, and by (b) $x$ is the unique point of $\partial \Omega$ such that $\left|x-y_{r}\right|=r$. Let $\tilde{x}=y_{r}+(1+\varepsilon)\left(x-y_{r}\right)$ for $\varepsilon \in\left(0, \frac{r}{2}\right)$. Given $\varepsilon$, there are two possibilities: either $\tilde{x} \in \Omega$ or $\tilde{x} \notin \Omega$.

Let $\tilde{x} \in \Omega$. Then, since $x \in \mathcal{R} \cap \partial \Omega, \quad \operatorname{dist}(\tilde{x}, \partial \Omega)<|x-\tilde{x}|=\varepsilon r$. Let $\tilde{y} \in \partial \Omega$ be such that $|\tilde{x}-\tilde{y}|=\operatorname{dist}(\tilde{x}, \partial \Omega)$, let $\tilde{y}_{r}=\tilde{y}+r \frac{\tilde{y}-\tilde{x}}{\mid \tilde{y}-\tilde{x}}$. Then $\tilde{y}_{r} \in \partial \Omega_{r}$ and by $(\mathrm{c})$ we get $\left|\tilde{x}-\tilde{y}_{r}\right|=\operatorname{dist}(\tilde{x}, \partial \Omega)+r=\operatorname{dist}\left(\tilde{x}, \partial \Omega_{r}\right)$, and $\operatorname{sodist}\left(\tilde{x}, \partial \Omega_{r}\right)<(\varepsilon+1) r=\left|\tilde{x}-y_{r}\right|$, so $y_{r} \notin \mathcal{N}_{\partial \Omega_{r}}(\tilde{x})$.

Let now $\tilde{x} \notin \Omega$, i.e., $\tilde{x} \in \Omega_{r} \backslash \bar{\Omega}$ since $\varepsilon<\frac{r}{2}$. Then, by (c), $\operatorname{dist}\left(\tilde{x}, \partial \Omega_{r}\right)<r$, but $\left|\tilde{x}-y_{r}\right|=(\varepsilon+1) r$, so $y_{r} \notin \mathcal{N}_{\partial \Omega_{r}}(\tilde{x})$.

Thus $y_{r} \notin \mathcal{N}_{\partial \Omega_{r}}(\tilde{x})$ for any small $\varepsilon>0$. This implies $x \in \mathcal{R}_{r}$.

Thus $\mathcal{R} \subset \mathcal{R}_{r}$.

Now we show that $\mathcal{R}_{r} \subset \mathcal{R}$. By (b), (c) we get $\mathcal{R}_{r} \cap\left(\bar{\Omega}_{r} \backslash \bar{\Omega}\right)=\emptyset$.

It remains to consider $x \in \bar{\Omega} \cap \mathcal{R}_{r}$. Let first $x \in \Omega \cap \mathcal{R}_{r}$. Let as above $y \in \mathcal{N}_{\partial \Omega}(x)$, $y_{r}=y+r \frac{y-x}{|y-x|} \in \mathcal{N}_{\partial \Omega_{r}}(x), \tilde{x}=y+(1+\varepsilon)(x-y)$ where $\varepsilon>0$ is small. From $x \in \mathcal{R}_{r}$ 
we conclude that $\operatorname{dist}\left(\tilde{x}, \partial \Omega_{r}\right)<\left|\tilde{x}-y_{r}\right|=(1+\varepsilon) \operatorname{dist}(x, \partial \Omega)+r$. Let $\tilde{y}_{r} \in \partial \Omega_{r}$ be such that $\left|\tilde{x}-\tilde{y}_{r}\right|=\operatorname{dist}\left(\tilde{x}, \partial \Omega_{r}\right)$ and let $\tilde{y}$ be the unique point of $\partial \Omega$ nearest to $\tilde{y}_{r}$, then $\left|\tilde{y}-\tilde{y}_{r}\right|=r$ (uniqueness of $\tilde{y}$ follows from (b)). Then by (c), (b) the points $\tilde{x}, \tilde{y}, \tilde{y}_{r}$ lie on one distance ray and $\operatorname{dist}(\tilde{x}, \partial \Omega)=|\tilde{x}-\tilde{y}|$, so

$$
\begin{gathered}
\operatorname{dist}(\tilde{x}, \partial \Omega)=|\tilde{x}-\tilde{y}|=\left|\tilde{y}_{r}-\tilde{x}\right|-\left|\tilde{y}_{r}-y\right|<\left|\tilde{x}-y_{r}\right|-r= \\
(1+\varepsilon) \operatorname{dist}(x, \partial \Omega)=(1+\varepsilon)|x-y|=|\tilde{x}-y|
\end{gathered}
$$

and so $\tilde{x} \in \mathcal{R}$.

The case of $x \in \partial \Omega \cap \mathcal{R}_{r}$ is similar to the case above.

Remark 10. Examples of nonconvex polygons on a plane show that without assuming some condition of the type of Condition 3 the statements (b)-(d) of Proposition 9 are not true in general.

Proposition 11. Let $\Omega \in R^{n}$ satisfy Condition $\Omega$ with radius $r$. Then $\partial \Omega_{\rho} \in C^{1,1}$ for any $\rho \in\left(0, \frac{r}{M}\right)$.

Proof. The assertion follows from inequality 4.8(8) of Fed59 and Proposition 9 (d). Also, inequality (96) proved in Appendix A1 can be applied instead of 4.8(8) of Fed59.

Recall the following facts (see e.g. GT83, Lemma 14.16, 14.17). Let $\Omega \subset \mathbf{R}^{n}$ be an open bounded set with $C^{2}$ boundary $\partial \Omega$. Let $\Omega_{r}$ be, as above, $r$-neighborhood of $\Omega$. Then $\partial \Omega_{r} \in C^{2}$ for small $r$. If $y \in \partial \Omega$ and if $y_{r} \in \partial \Omega_{r}$ is the unique point on $\partial \Omega_{r}$ such that $\left|y-y_{r}\right|=r$ then the principal coordinate systems of $\partial \Omega$ at $y$ and of $\partial \Omega_{r}$ at $y_{r}$ are parallel. Denote by $\kappa_{i}$ and $\kappa_{i, r}, \quad(i=1, \ldots n-1)$ the principal curvatures of $\partial \Omega$ at $y$ and of $\partial \Omega_{r}$ at $y_{r}$ respectively. We have

$$
\kappa_{i, r}=\frac{\kappa_{i}}{1+\kappa_{i} r} \quad \text { for } \quad i=1, \ldots, n-1 .
$$

In the next two propositions we show similar facts for sets with nonsmooth boundaries satisfying Condition 3 and for Lipschitz families of such sets.

Proposition 12. Let $\Omega \subset \mathbf{R}^{n}$ be an open set satisfying Condition 3 with radius $r_{0}$. Let $0<r \leq r_{0}$. Fix $y \in \partial \Omega$. Let $y_{r} \in \partial \Omega_{r}$ be such point that $\left|y-y_{r}\right|=r$. Then:

a) Let $\partial \Omega$ be twice differentiable at $y$, i.e., in a suitable coordinate system in $\mathbf{R}^{n}$ we have $y=0, \quad \Omega \cap B_{\delta}(0)=\left\{x_{n}>\Psi\left(x^{\prime}\right)\right\} \cap B_{\delta}(0)$ for small $\delta>0$ and $\Psi\left(x^{\prime}\right)=$ $\sum_{i=1}^{n-1} \kappa_{i} x_{i}^{2}+o\left(\left|x^{\prime}\right|^{2}\right)$. Then the point $y_{r}$ is unique given the point $y$, and in the coordinate system introduced above $y_{r}=(0,-r), \Omega_{r} \cap B_{\delta_{1}}\left(y_{r}\right)=\left\{x_{n}>\Psi_{r}\left(x^{\prime}\right)\right\} \cap$ $B_{\delta_{1}}\left(y_{r}\right)$ for sufficiently small $\delta_{1}>0$, where $\Psi_{r}\left(x^{\prime}\right)=-r+\sum_{i=1}^{n-1} \frac{\kappa_{i}}{1+\kappa_{i} r} x_{i}^{2}+o\left(\left|x^{\prime}\right|^{2}\right)$, i.e., $\Omega_{r}$ is twice differentiable at $y_{r}$ and (23) holds.

b) If $\partial \Omega_{r}$ is twice differentiable at $y_{r}$ and $\gamma_{\Omega}(y)>0$ then $\partial \Omega$ is twice differentiable at $y$ and (23) holds (i.e., the functions $\Psi$ and $\Psi_{r}$ have same expansions at 0 as in a)). 
Proof. a) Uniqueness of $y_{r}$ and the fact that $y_{r}=(0,-r)$ follow from smoothness of $\partial \Omega$ at $y$. So the interval connecting $y$ and $y_{r}$ lies on $x_{n}$-axis. By Propositions 9 and 11 the surface $\partial \Omega_{r}$ is of class $C^{1,1}$, and the interval connecting $y$ and $y_{r}$ is orthogonal to $\partial \Omega_{r}$. So $\Omega_{r} \cap B_{\delta_{1}}\left(y_{r}\right)=\left\{x_{n}>\Psi_{r}\left(x^{\prime}\right)\right\} \cap B_{\delta_{1}}\left(y_{r}\right)$ for some $\delta_{1}>0$, where $\Psi_{r}$ is a $C^{1,1}$ function.

Let $\varepsilon>0$. There exists $\sigma>0$ such that

$$
\left|\Psi\left(x^{\prime}\right)-\sum_{i=1}^{n-1} \kappa_{i} x_{i}^{2}\right|<\varepsilon\left|x^{\prime}\right|^{2} \text { if }\left|x^{\prime}\right|<\sigma .
$$

Denote $A_{\varepsilon}=\left\{\left(z^{\prime}, z_{n}\right) \in \mathbf{R}^{n} \mid z_{n} \geq \sum_{i=1}^{n-1}\left(\kappa_{i}-\varepsilon\right) z_{i}^{2}\right\}$. Then we get:

$$
\partial \Omega \cap B_{\sigma}(0) \subset A_{\varepsilon} \backslash A_{-\varepsilon} .
$$

Since $\gamma_{\mathbf{R}^{n} \backslash \bar{\Omega}}(y) \geq 2 r$, the point $y_{r}$ lies in the relative interior of the distance ray $R_{y}$ of the set $\mathbf{R}^{n} \backslash \bar{\Omega}$. Then there exists $\sigma_{1}>0$ such that for any point $x_{r} \in \cap B_{\sigma_{1}}\left(y_{r}\right)$ we have $\mathcal{N}_{\partial \Omega}\left(x_{r}\right) \subset \partial \Omega \cap B_{\sigma}(0)$. (Proof: suppose this is false, then there exist $\sigma_{1}>0$ and a sequence $x_{r}^{k} \rightarrow y_{r}$ such that for $x^{k} \in \mathcal{N}_{\partial \Omega}\left(x_{r}^{k}\right)$ we get $\left|x^{k}-y\right|>\sigma_{1}$. Then, passing to a subsequence, we get $x^{k} \rightarrow x$ where $x \in \partial \Omega$ and $x \neq y$. By continuity of distance function we get $x \in \mathcal{N}_{\partial \Omega}\left(y_{r}\right)$ and $x \neq y$. By Condition $3 y_{r}$ lies in the relative interior of the distance ray $R_{y}$ of the set $\mathbf{R}^{n} \backslash \bar{\Omega}$. Thus $y_{r}$ has a unique nearest point on $\partial \Omega$. A contradiction.). So

$$
x \in A_{\varepsilon} \backslash A_{-\varepsilon} \quad \text { if } \quad x \in \mathcal{N}_{\partial \Omega}\left(x_{r}\right) \quad \text { where } \quad x_{r} \in B_{\sigma_{1}}\left(y_{r}\right) .
$$

Let $N_{\varepsilon}$ be $r$-neighborhood of the set $A_{\varepsilon}$. By Remark 4 we have $\kappa_{i} \geq-\frac{1}{2 r}$. Let $\varepsilon<\frac{1}{4 r}$. Then (by GT83 Lemma 14.16,14.17), $\partial N_{\varepsilon}$ is the graph of a $C^{\infty}$ function $g_{\varepsilon}$, and $\frac{\partial^{2} g_{\varepsilon}}{\partial x_{i} \partial x_{j}}(0)=\frac{\kappa_{i}-\varepsilon}{1+\left(\kappa_{i}-\varepsilon\right) r} \delta_{i j}$. Let

$$
\tilde{N}_{\varepsilon, \delta}=\left\{\left(z^{\prime}, z_{n}\right) \mid z_{n} \geq \sum_{i=1}^{n-1}\left(\frac{\kappa_{i}-\varepsilon}{1+\left(\kappa_{i}-\varepsilon\right) r}-\delta\right) z_{1}^{2}-r\right\} .
$$

If $\sigma_{2}>0$ is small enough then

$$
\tilde{N}_{ \pm \varepsilon,-\varepsilon} \cap B_{\sigma_{2}}\left(y_{r}\right) \subset N_{ \pm \varepsilon} \cap B_{\sigma_{2}}\left(y_{r}\right) \subset \tilde{N}_{ \pm \varepsilon, \varepsilon} \cap B_{\sigma_{2}}\left(y_{r}\right) .
$$

Thus if we choose $\sigma_{3}$ small, then we get by (24), (25)

$$
\partial \Omega_{r} \cap B_{\sigma_{3}}\left(y_{r}\right) \subset \tilde{N}_{\varepsilon, \varepsilon} \backslash \tilde{N}_{-\varepsilon,-\varepsilon},
$$

which means that for $x_{r}=\left(x_{r}^{\prime}, \Psi_{r}\left(x_{r}^{\prime}\right)\right) \in \partial \Omega_{r} \cap B_{\sigma_{3}}\left(y_{r}\right)$ we have

$$
\sum_{i=1}^{n-1}\left(\frac{\kappa_{i}-\varepsilon}{1+\left(\kappa_{i}-\varepsilon\right) r}-\varepsilon\right) x_{r, i}^{2}-r \leq \Psi_{r}\left(x_{r}^{\prime}\right) \leq \sum_{i=1}^{n-1}\left(\frac{\kappa_{i}+\varepsilon}{1+\left(\kappa_{i}+\varepsilon\right) r}+\varepsilon\right) x_{r, i}^{2}-r .
$$

Sending $\varepsilon$ to 0 we conclude the proof.

b) By Proposition 9 the condition $\gamma_{\Omega}(y)>0$ implies that $\gamma_{\Omega_{r}}\left(y_{r}\right)>r$. Now we can perform calculation similar to the proof of assertion a). 
We need similar facts for families of sets. Let $\left\{\Omega_{t}\right\}$ be a family of open sets in $\mathbf{R}^{n}$. Let $\left(\Omega_{t}\right)_{r}$ be $r$-neighborhood of $\Omega_{t}$ in $\mathbf{R}^{n}$. Define

$$
E_{r}=\cup_{t}\left[\left(\Omega_{t}\right)_{r} \times\{t\}\right], \quad \Gamma_{r}=\cup_{t}\left[\partial\left(\Omega_{t}\right)_{r} \times\{t\}\right] .
$$

Proposition 13. Let $\left\{\Omega_{t}\right\}$ be a locally Lipschitz continuous family of open sets in $\mathbf{R}^{n}$. Let for every $t \in \mathbf{R}_{+}^{1}$ the set $\Omega_{t}$ satisfy Condition $\Omega$ with radius $r_{0}>0$. Let $0<r \leq r_{0}$. Fix $y \in \partial \Omega_{t_{0}}$. Let $y_{r} \in \partial\left(\Omega_{t_{0}}\right)_{r}$ be such point that $\left|y-y_{r}\right|=r$. Then:

a) Let $\Gamma$, defined by (5), be (2,1) differentiable at $\left(y, t_{0}\right)$. That is in a suitable coordinate system $\left(x_{1}, \ldots, x_{n}\right)$ in $\mathbf{R}^{n}$ we have

$$
y=0, \Omega \cap B_{\delta}^{n+1}(0)=\left\{x_{n}>\Psi\left(x^{\prime}, t\right)\right\} \cap B_{\delta}^{n+1}(0)
$$

for small $\delta>0$ and

$$
\Psi\left(x^{\prime}, t\right)=v\left(t-t_{0}\right)+\sum_{i=1}^{n-1} \kappa_{i} x_{1}^{2}+o\left(\left|t-t_{0}\right|+\left|x^{\prime}\right|^{2}\right) .
$$

Then the point $y_{r}$ is unique given the point $y$, and in the coordinate system introduced above $y_{r}=(0,-r)$,

$$
\Omega_{r} \cap B_{\delta_{1}}^{n+1}\left(y_{r}\right)=\left\{x_{n}>\Psi_{r}\left(x^{\prime}, t\right)\right\} \cap B_{\delta_{1}}^{n+1}\left(y_{r}\right)
$$

for sufficiently small $\delta_{1}>0$, where

$$
\Psi_{r}\left(x^{\prime}, t\right)=-r+v\left(t-t_{0}\right)+\sum_{i=1}^{n-1} \frac{\kappa_{i}}{1+\kappa_{i} r} x_{1}^{2}+o\left(\left|t-t_{0}\right|+\left|x^{\prime}\right|^{2}\right) .
$$

b) If $\Gamma_{r}$ is (2,1) differentiable at $\left(y_{r}, t_{0}\right)$ and $\gamma_{\Omega_{t_{0}}}(y)>0$ then $\Gamma$ is $(2,1)$ differentiable at $y$ and the functions $\psi$ and $\psi_{r}$ have same expansions at $\left(0, t_{0}\right)$ as in a).

Proof. The proof is similar to the one of Proposition 12. In particular, the sets $A_{\varepsilon}$, $N_{\varepsilon}$ and $\tilde{N}_{\varepsilon, \delta}$ are defined as following.

$$
A_{\varepsilon}=\left\{\left(z^{\prime}, z_{n}, t\right)\left|z_{n} \geq v\left(t-t_{0}\right)+\sum_{i=1}^{n-1}\left(\kappa_{i}-\varepsilon\right) z_{i}^{2}-\varepsilon\right| t-t_{0} \mid\right\} .
$$

$N_{\varepsilon}$ is defined as following: for each $t=t^{*}$ the set $N_{\varepsilon} \cap\left\{t=t^{*}\right\}$ is $\varepsilon$-neighborhood in $\mathbf{R}^{n}$ of the set $\left\{x \mid\left(x, t^{*}\right) \in A_{\varepsilon}\right\}$. Finally,

$$
\tilde{N}_{\varepsilon, \delta}=\left\{\left(z^{\prime}, z_{n}\right)\left|z_{n} \geq v\left(t-t_{0}\right)+\sum_{i=1}^{n-1}\left(\frac{\kappa_{i}-\varepsilon}{1+\left(\kappa_{i}-\varepsilon\right) r}-\delta\right) z_{1}^{2}-r-(\varepsilon+\delta)\right| t-t_{0} \mid\right\} .
$$

The rest of the argument does not change.

To derive further properties of the distance function of a set $\Omega$ satisfying the Condition 3, we need to integrate over such sets. To do this it is convenient to decompose the set by suitable subsets, and define a local coordinate system in each subset. The construction is carried out below and can be roughly described as following. The subsets are unions of distance rays passing through subsets of the boundary. The coordinate systems consist of variables $x_{1}, \ldots, x_{n-1}$ on the boundary, and the variable 
$x_{n}$ along the distance rays. However, since $\partial \Omega$ is not smooth enough, we have to use $\partial \Omega_{r}$. Now we turn to the construction.

By Proposition 11 the set $\Omega_{r}$ for small enough $r>0$ has $C^{1,1}$ boundary. Fix such $r$. We can choose sets $\mathcal{U}_{1}, \ldots, \mathcal{U}_{N}$, bounded and open in $\mathbf{R}^{n}$, so that $\partial \Omega_{r} \subset \cup_{k=1}^{N} \mathcal{U}_{k}$, and for each $k=1, \ldots, N$ in a suitable coordinate system in $\mathbf{R}^{n}$ we have

$$
\Omega_{r} \cap \mathcal{U}_{k}=\left\{\left(x^{\prime}, x_{n}\right) \mid x^{\prime} \in \tilde{U}_{k}, x_{n}>\Phi_{k}\left(x^{\prime}\right)\right\} \cap \mathcal{U}_{k},
$$

where $\tilde{U}_{k}=\left\{x^{\prime} \mid\left(x^{\prime}, x_{n}\right) \in \mathcal{U}_{k}\right\} \subset \mathbf{R}^{n-1}$ is an open set, and $\Phi_{k}$ is a $C^{1,1}$ function on $\mathbf{R}^{n-1}$.

We can also choose open sets $\mathcal{U}_{0}$ and $\mathcal{U}_{N+1} \subset \mathbf{R}^{n}$ such that $\mathcal{U}_{0} \subset \Omega_{r}, \mathcal{U}_{N+1} \subset$ $\mathbf{R}^{n} \backslash \Omega_{r}$, both $\mathcal{U}_{0}$ and $\mathcal{U}_{N+1}$ do not intersect $\partial \Omega$, and $\mathbf{R}^{n}=\cup_{k=0}^{N+1} \mathcal{U}_{k}$. Let $\tilde{\Psi}_{k}, k=$ $1, \ldots, N$, be a smooth partition of unity on $\mathbf{R}^{n}$ related to the sets $\mathcal{U}_{0}, \mathcal{U}_{1}, \ldots, \mathcal{U}_{N+1}$, i.e., $\sum_{k=0}^{N+1} \tilde{\Psi}_{k} \equiv 1$ on $\mathbf{R}^{n}$ and $\tilde{\Psi}_{k} \in C_{0}^{\infty}\left(\mathcal{U}_{k}\right)$. Then by our choice of $\mathcal{U}_{0}$ and $\mathcal{U}_{N+1}$ we get $\sum_{k=1}^{N} \tilde{\Psi}_{k} \equiv 1$ on $\partial \Omega_{r}$.

Define maps $G_{k}: \tilde{U}_{k} \times \mathbf{R}^{1} \rightarrow \mathbf{R}^{n}(k=1, \ldots, N)$ by

$$
G_{k}\left(x^{\prime}, x_{n}\right)=y+x_{n} D d_{\Omega_{r}}^{s}(y)
$$

where $y=\left(x^{\prime}, \Phi_{k}\left(x^{\prime}\right)\right) \in \partial \Omega_{r}$, and $d_{\Omega_{r}}^{s}(\cdot)$ is the signed distance to $\partial \Omega_{r}$.

For each $z \in \partial \Omega_{r}$ the vector $D d_{\Omega_{r}}^{s}(z)$ is the inner unit normal to $\partial \Omega_{r}$ in $z$. Since $\partial \Omega_{r}$ is a $C^{1,1}$ manifold, $D d_{\Omega_{r}}^{s}$ is Lipschitz on $\partial \Omega_{r}$. Then since $\Phi_{k}$ is $C^{1,1}$ on $\mathbf{R}^{n-1}$, the map $G_{k}$ is Lipschitz on bounded subsets of $\tilde{U}_{k} \times \mathbf{R}^{1}$. In particular, the map $\tilde{U}_{k} \rightarrow \partial \Omega_{r}$ defined by $x^{\prime} \rightarrow G_{k}\left(x^{\prime}, 0\right)$ is Lipschitz.

From that by explicit computation we get the following

Lemma 14. Let the map $G_{k}: \tilde{U}_{k} \times \mathbf{R}^{1} \rightarrow \mathbf{R}^{n}$ be defined by (2X). Then the Jacobian $J G_{k}$ is a locally bounded measurable function. For $\mathcal{L}^{n-1}$ a.e. point $x^{\prime} \in \tilde{U}_{k}$ the surface $\partial \Omega_{r}$ is twice differentiable at the point $G_{k}\left(x^{\prime}, 0\right)$. For every such point $x^{\prime}$ the map $G_{k}$ is differentiable at $\left(x^{\prime}, x_{n}\right)$ for every $x_{n} \in \mathbf{R}^{1}$. At every such point $\left(x^{\prime}, x_{n}\right)$ the Jacobian of $G_{k}$ is given by

$$
J_{n} G_{k}\left(x^{\prime}, x_{n}\right)=\sqrt{1+\left|D \Phi_{k}\left(x^{\prime}\right)\right|^{2}} \prod_{i=1}^{n-1}\left[1-\kappa_{r, i} x_{n}\right],
$$

where $\kappa_{r, 1}, \ldots, \kappa_{r, n-1}$ are the principal curvatures of $\partial \Omega_{r}$ at $G_{k}\left(x^{\prime}, 0\right)$.

Denote by $U_{k}$ the sets

$$
U_{k}=\cup_{x^{\prime} \in \tilde{U}_{k}}\left\{\left(x^{\prime}, x_{n}\right) \mid x_{n} \in\left[0, \gamma_{\Omega_{r}}(y)\right)\right\} \subset \mathbf{R}^{n},
$$

where $\gamma_{\Omega_{r}}: \mathbf{R}^{n} \rightarrow \mathbf{R}^{1}$ is the ridge function (21) of the set $\Omega_{r}$. Note that $\gamma_{\Omega_{r}}(z) \geq r$ for all $z \in \bar{\Omega}_{r}$ by Proposition 9 (c, d).

The map $G_{k}$ is one-to-one on $U_{k}$ (by definition of $\gamma_{\Omega_{r}}$ and Lemma 3.4 of [EH87]). In addition $\Omega \backslash \mathcal{R} \subset \cup_{k=1}^{N} G_{k}\left(U_{k}\right)$, since every point of $\Omega_{r} \backslash \mathcal{R}$ lies inside some distance ray. For each $k=1, \ldots, N$ define a function $\Psi_{k}: \mathbf{R}^{n} \rightarrow \mathbf{R}^{1}$ by extending the function $\tilde{\Psi}_{k}$ from the boundary inside $\Omega_{r}$ as a constant along distance rays and defining $\Psi_{k}$ 
to be the 0 on the ridge of $\Omega_{r}$ and outside $\Omega_{r}$, i.e.,

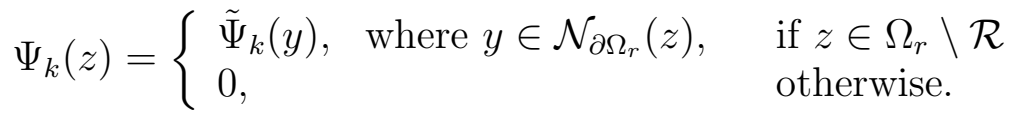

Note that $y$ is uniquely defuned by $z$ in the first case of (30). We have

$$
\operatorname{supp}_{k} \subset G_{k}\left(U_{k}\right) ; \quad \sum_{k=1}^{N} \tilde{\Psi}_{k}(y(z)) \equiv 1 \text { for all } z \in \Omega_{r} \backslash \mathcal{R} .
$$

We thus decomposed the set $\Omega_{r} \backslash \mathcal{R}$ by the sets $G_{k}\left(U_{k}\right)$ and defined a related partition of unity $\Psi_{k}$. Note that in general the sets $U_{k}$ and $G_{k}\left(U_{k}\right)$ are neither open nor closed, and the functions $\Psi_{k}$ are discontinuous. In the next propositions we prove that these sets and functions are measurable.

Proposition 15. Define a function $\tilde{\gamma}_{r, k}: \mathbf{R}^{n-1} \rightarrow \mathbf{R}^{1}$ by

$$
\tilde{\gamma}_{r, k}\left(x^{\prime}\right)= \begin{cases}\gamma_{\Omega_{r}}\left(G_{k}\left(x^{\prime}, 0\right)\right), & \text { if } x^{\prime} \in \operatorname{cl}\left(\tilde{U}_{k}\right) \\ 0, & \text { if } x^{\prime} \notin \operatorname{cl}\left(\tilde{U}_{k}\right)\end{cases}
$$

where $\operatorname{cl}(\cdot)$ denotes closure of a set. Then $\tilde{\gamma}_{r, k}$ is uppersemicontinuous (usc). The sets $U_{k}$ are $\mathcal{L}^{n}$ measurable. The ridge set of $\Omega_{r}$ has $\mathcal{L}^{n}$ measure 0.

Proof. It is enough to show that for any sequence $\left\{x_{m}^{\prime}\right\}_{m=1}^{\infty}$ such that $x_{m}^{\prime} \in \operatorname{cl}\left(\tilde{U}_{k}\right)$, $x_{m}^{\prime} \rightarrow x^{\prime}$ and $\tilde{\gamma}_{r, k}\left(x_{m}^{\prime}\right) \rightarrow L$ we have $\gamma_{r, k}\left(x^{\prime}\right) \geq L$.

The function $\gamma_{\Omega_{r}}$ is usc on $\mathbf{R}^{n}$. Since $x \in \operatorname{cl}\left(\tilde{U}_{k}\right)$ and $G_{k}$ is Lipschitz on $\left.\operatorname{cl}\left(\tilde{U}_{k}\right) \times 0\right)$,

$$
\tilde{\gamma}_{r, k}\left(x^{\prime}\right)=\gamma_{\Omega_{r}}\left(G_{k}\left(x^{\prime}, 0\right)\right) \geq \lim _{m \rightarrow \infty} \gamma_{\Omega_{r}}\left(G_{k}\left(\left(x_{m}^{\prime}\right)\right)\right.
$$

Thus, $\tilde{\gamma}_{r, k}$ is usc.

We have $U_{k}=\left\{\left(x^{\prime}, x_{n}\right) \mid x^{\prime} \in \tilde{U}_{k}, 0 \leq x_{n}<\tilde{\gamma}_{r, k}\left(x^{\prime}\right)\right\}$. Thus $U_{k}$ is $\mathcal{L}^{n}$-measurable (note that $\tilde{U}_{k}$ is open in $\mathbf{R}^{n-1}$ ).

To prove that the ridge has $\mathcal{L}^{n}$ measure 0 , we note that $G_{k}^{-1}(\mathcal{R}) \cap \bar{U}_{k}=\left\{\left(x^{\prime}, x_{n}\right) \mid x^{\prime} \in\right.$ $\left.\tilde{U}_{k}, x_{n}=\tilde{\gamma}_{r, k}\left(x^{\prime}\right)\right\}$. Then $\mathcal{L}^{n}\left(G_{k}^{-1}(\mathcal{R}) \cap \bar{U}_{k}\right)=0$ because $\tilde{\gamma}_{r, k}(\cdot)$ is usc. Since $G_{k}$ is Lipschitz on $\operatorname{cl}\left(\tilde{U}_{k}\right) \times\left[0, \operatorname{diam} \Omega_{r}\right]$ and since $\cup_{k=1}^{N} \bar{U}_{k}=\bar{\Omega}_{r}$, we have $\mathcal{L}^{n}(\mathcal{R})=0$.

We will use repeatedly the following fact:

Lemma 16. Let $f: A \subset \mathbf{R}^{m} \rightarrow \mathbf{R}^{n}(m \leq n)$ be a Lipschitz function, $A$ be a $\mathcal{L}^{m}$ measurable set, and let $g: \mathbf{R}^{m} \rightarrow \mathbf{R}^{1}$ be a $\mathcal{L}^{m}$-measurable function. Then the function $g \circ f^{-1}: \mathbf{R}^{n} \rightarrow \mathbf{R}^{1}$ is $\mathcal{L}^{n}$-measurable.

Proof. We can assume that $g \equiv 0$ outside $A$. Let $V \subset \mathbf{R}^{1} \cup\{+\infty\}$ be an open set. Then $W=g^{-1}(V) \cap A$ is $\mathcal{L}^{m}$-measurable. Then $\left(g \circ f^{-1}\right)^{-1} V=f\left(g^{-1}(U)\right) \cap A=$ $f(W)$, which is a measurable set since $f$ is Lipschitz and $m \leq n$ (see [EGar92, Lemma 2 of 3.3.1).

Proposition 17. Functions $\Psi_{k}$ and $\Psi_{k} \circ G_{k}$ are $\mathcal{L}^{n}$-measurable. 
Proof. The functions $G_{k}$ are Lipschitz. Then by Lemma 16 it is enough to prove that functions $\Psi_{k} \circ G_{k}$ are $\mathcal{L}^{n}$-measurable (since then $\left.\Psi_{k}=\left(\Psi_{k} \circ G_{k}\right) \circ G_{k}^{-1}\right)$. Let $\left(x^{\prime}, x_{n}\right) \in U_{k}$. Then

$$
\Psi_{k}\left(G_{k}\left(x^{\prime}, x_{n}\right)\right)=\tilde{\Psi}_{k}\left(G_{k}\left(x^{\prime}, 0\right)\right)=\tilde{\Psi}_{k}\left(x^{\prime}, \Phi_{k}\left(x^{\prime}\right)\right) .
$$

Consider the function $\Theta\left(x^{\prime}, x_{n}\right)=\tilde{\Psi}_{k}\left(x^{\prime}, \Phi_{k}\left(x^{\prime}\right)\right)$. Since $\tilde{\Psi}_{k} \in C^{\infty}\left(\mathbf{R}^{n}\right)$ and $\Phi_{k} \in$ $C^{1,1}\left(\mathbf{R}^{n-1}\right)$, we see that $\Theta$ is a $C^{1,1}$ function $\mathbf{R}^{n} \rightarrow \mathbf{R}^{1}$. Now since $\Psi_{k} \circ G_{k}=\Theta$ on $U_{k}, \Psi_{k} \circ G_{k} \equiv 0$ outside $U_{k}$, and by Proposition 15 the set $U_{k}$ is measurable, the assertion follows.

Proposition 18. Let $\Omega$ be a bounded open set satisfying the Condition S. Let $X \subset$ $\partial \Omega$ and $\mathcal{H}^{n-1}(X)=0$. Let $Y$ be the union of distance rays passing through $X$, i.e., $Y=\cup_{x \in X} R_{x}$. Then $\mathcal{L}^{n}(Y)=0$.

Proof. We can assume that $X \subset G_{k}\left(U_{k}\right) \cap \partial \Omega$ for some $k$, and then $Y \subset G_{k}\left(U_{k}\right)$. We also can assume that

$$
\gamma_{\Omega}(x)>0 \text { for all } x \in X .
$$

If (32) is not true then we can replace $X$ by $X \cap\left\{x \mid \gamma_{\Omega}(x)>0\right\}$ and $Y$ does not change.

Since $\tilde{\gamma}_{r, k}\left(x^{\prime}\right)$ is a u.s.c. function of $x^{\prime}$, we get $\mathcal{L}^{n}\left(\left\{x_{n}=\gamma_{r, k}\left(x^{\prime}, 0\right)\right\}\right)=0$. We also know that $J_{n} G_{k}>0$ on the set $U_{k}$. The map $G_{k}$ is one-to-one on $U_{k}$, and so $N\left(G_{k}, y\right)=1$ for $y \in Y \subset G_{k}\left(U_{k}\right)$, where $N(f, z)$ denotes the multiplicity function, which is the number of elements of $f^{-1}(z)$. Then by area formula we obtain

$$
\mathcal{L}^{n}(Y)=\int_{\mathbf{R}^{n}} \chi_{Y}(y) d y=\int_{U_{k}} \chi_{Y}\left(G_{k}(x)\right) J_{n} G_{k}(x) d x,
$$

where $\chi_{A}(\cdot)$ is the characteristic function of the set $A$, i.e., $\chi_{A}(\cdot)$ equals 1 on $A$ and 0 outside $A$. By Lemma 14 the Jacobian $J_{n} G_{k}$ is a locally bounded measurable function. Let $x^{\prime} \in \tilde{U}_{k}$ be such point that the surface $\partial \Omega_{r}$ is twice differentiable at the point $G_{k}\left(x^{\prime}, 0\right)$. By Lemma 14 the Jacobian $J_{n} G_{k}$ is defined by the expression (28) at $\left(x^{\prime}, x_{n}\right)$ for every $x_{n} \in \mathbf{R}^{1}$. The principal curvatures of $\partial \Omega_{r}$ at the point $G_{k}\left(x^{\prime}, 0\right)$ satisfy

$$
\kappa_{r, i}>-\frac{1}{r}, \quad \kappa_{r, i} \tilde{\gamma}_{r, k}\left(x^{\prime}\right) \leq 1
$$

by definition of $\tilde{\gamma}_{r, k}(\cdot)$, and by Condition 3. We also have

$$
\tilde{\gamma}_{r, k}\left(x^{\prime}\right) \leq \operatorname{diam} \Omega_{r}
$$

Let $m \leq n$ be such that $-\frac{1}{r} \leq \kappa_{r, 1}, \ldots, \kappa_{r, m}<0$ and $\kappa_{r, m+1}, \ldots, \kappa_{r, n-1} \geq 0$. Then for $x_{n} \in\left(r, \tilde{\gamma}_{r, k}\left(x^{\prime}\right)\right)$ we calculate using (34), (35):

$$
0 \leq \frac{J_{n} G_{k}\left(x^{\prime}, x_{n}\right)}{J_{n} G_{k}\left(x^{\prime}, r\right)} \leq \frac{\prod_{i=1}^{m}\left[1-\kappa_{r, i} x_{n}\right]}{\prod_{i=1}^{m}\left[1-\kappa_{r, i} r\right]} \leq\left[1+\frac{1}{r} \tilde{\gamma}_{r, k}\left(x^{\prime}\right)\right]^{m} \leq C,
$$

where $C$ depends on $r, n$ and $\operatorname{diam}(\Omega)$. By Lemma 14 the inequalities (36) hold for a.e. $x \in U_{k} \cap\left\{\left(x^{\prime}, x_{n}\right) \mid x_{n}>r\right\}$. 
Define a map $\tilde{G}_{k}: \tilde{U}_{k} \rightarrow \partial \Omega \subset \mathbf{R}^{n}$ by $\tilde{G}_{k}\left(x^{\prime}\right)=G_{k}\left(x^{\prime}, r\right)$. Then by (28)

$$
J_{n-1} \tilde{G}_{k}\left(x^{\prime}\right)=J_{n} G_{k}\left(x^{\prime}, r\right) \text { for a.e. } x^{\prime} \in \tilde{U}_{k} \text {. }
$$

So using the fact that the relation $Y \subset \bar{\Omega}$ implies $\chi_{Y}\left(G_{k}\left(x^{\prime}, x_{n}\right)\right)=0$ for $x_{n}<r$, we get from (33), (35), (36) and (37) using area formula:

$$
\begin{aligned}
\mathcal{L}^{n}(Y) & \leq C \int_{U_{k}} \chi_{Y}\left(G_{k}(x)\right) J_{n-1} \tilde{G}_{k}\left(x^{\prime}\right) d x \\
& =C \int_{\tilde{U}_{k}} J_{n-1} \tilde{G}_{k}\left(x^{\prime}\right)\left[\int_{r}^{\tilde{\gamma}_{r, k}\left(x^{\prime}\right)} \chi_{Y}\left(G_{k}\left(x^{\prime}, x_{n}\right)\right) d x_{n}\right] d x^{\prime} \\
& =C \int_{\tilde{U}_{k}} \chi_{X}\left(\tilde{G}_{k}\left(x^{\prime}\right)\right)\left[\tilde{\gamma}_{r, k}\left(x^{\prime}\right)-r\right] \cdot J_{n-1} \tilde{G}_{k}\left(x^{\prime}\right) d x^{\prime} \\
& \leq C \int_{\tilde{U}_{k}} \chi_{X}\left(\tilde{G}_{k}\left(x^{\prime}\right)\right) J_{n-1} \tilde{G}\left(x^{\prime}\right) d x^{\prime} \\
& =C \int_{\partial \Omega} \chi_{X}(z) N\left(\tilde{G}_{k}, z\right) d \mathcal{H}^{n-1}(z)=C \mathcal{H}^{n-1}(X)=0
\end{aligned}
$$

where we have used the fact that $N\left(\tilde{G}_{k}, z\right)=1$ for $z \in X$ (which follows from (32) and Proposition $9(\mathrm{~b}, \mathrm{c}))$.

Remark 19. Without assuming a condition of the type of Condition 8 the assertion of Proposition 18 is not true. For example consider a nonconvex polygon in $R^{2}$ and take $X$ to be one point, a vertex of a re-enterant corner.

Remark 20. Note that it follows from the proof above that

$$
\mathcal{L}^{n}\left(G_{k}^{-1}(Y)\right)=\int_{\tilde{U}_{k}} J_{n-1} \tilde{G}_{k}\left(x^{\prime}\right)\left[\int_{r}^{\tilde{\gamma}_{r, k}\left(x^{\prime}\right)} \chi_{Y}\left(G_{k}\left(x^{\prime}, x_{n}\right)\right) d x_{n}\right] d x^{\prime}=0 .
$$

The following proposition describes the structure of the boundary of a set that satisfies Condition 3 .

Proposition 21. Let $\Omega$ be a bounded set. Let $\Omega$ satisfy the condition 3 with radius $r_{0}$. Then $\partial \Omega$ is $\left(\mathcal{H}^{n-1}, n-1\right)$ rectifiable subset of $\mathbf{R}^{n}$. In addition, $\partial \Omega$ is twice differentiable $\mathcal{H}^{n-1}$ a.e. on the set

$$
\mathcal{B}=\left\{x \in \partial \Omega \mid \gamma_{\Omega}(x)>0\right\} .
$$

Proof. Let $r>0$ be such number that $\partial \Omega_{r}$ is $C^{1,1}$. Existence of such $r$ follows from Proposition 11. It follows from Proposition 9 b) that the nearest point projection mapping $P: \Omega_{r} \rightarrow \Omega$ is well-defined and onto. Moreover, by inequality 4.8(8) of Fed59 (or by inequality (95) proved in Appendix A below) the map $P$ is Lipschitz. Since $\partial \Omega_{r}$ is $C^{1,1}$, we get that $\partial \Omega$ is $\left(\mathcal{H}^{n-1}, n-1\right)$ rectifiable.

By Proposition 9 every point of the set $\mathcal{B}$ has a unique nearest point on $\partial \Omega_{r}$. Since $\partial \Omega_{r}$ is $C^{1,1}$, it follows that $\partial \Omega_{r}$ is twice differentiable $\mathcal{H}^{n-1}$ a.e. Since the map $P$ is Lipschitz it follows that for $\mathcal{H}^{n-1}$ a.e. $y \in \mathcal{B}$ the surface $\partial \Omega_{r}$ is twice differentiable at the corresponding point $y_{r}$. Applying Proposition $12 \mathrm{~b}$ ), we conclude the proof. 
Propositions 15, 18 and 21 imply

Corollary 22. Let $\Omega \subset \mathbf{R}^{n}$ satisfy Condition 3 . Then $\mathcal{L}^{n}$ a.e point of $\Omega$ lies in the relative interior of a distance ray that intersects $\partial \Omega$ at a point at which $\partial \Omega$ is twice differentiable.

We need an analogue of the Proposition 21 for a Lipschitz family $\left\{\Omega_{t}\right\}$ of sets that satisfy Condition 3 with a radius $r_{0}$ independent of $t$. Let $E, \Gamma$ be defined by (5). Let $E_{r}, \Gamma_{r}$ be defined by (26). Denote

$$
D(x, t)=d_{\Omega_{t}}^{s}(x)
$$

where $d_{\Omega_{t}}^{s}(x)$ is the signed distance function (2).

Proposition 23. Let $\left\{\Omega_{t}\right\}$ be a locally Lipschitz family of open bounded sets that satisfy the Condition 5 with radius $r_{0}$ independent of $t$. Then

a) $\mathcal{L}^{n+1}$ a.e. $\left(x_{0}, t_{0}\right) \in E$ lies in the relative interior of a distance ray of the set $\Omega_{t_{0}}$ that intersects $\partial \Omega_{t_{0}}$ at such point $y \in \partial \Omega_{t_{0}}$ that $\Gamma$ is $(2,1)$ differentiable at $\left(y, t_{0}\right)$;

b) There exists a constant $C$ depending only on $r_{0}$ such that the function $D(x, t)-$ $C|x|^{2}$ on the set $E_{r_{0}}$ is concave in $x$ for every $t$.

Proof. First we prove the following fact.

Lemma 24. Let $\Omega$ satisfies Condition 3 with radius $r_{0}$. Then the distance function $d_{\Omega}(x)$ is semiconcave in $\Omega$, i.e. there exists a constant $C$ depending only on $r_{0}$ such that the function $d_{\Omega}(x)-C|x|^{2}$ is concave in $\Omega$.

Proof. Let $x \in \Omega$. Let $w \in \mathbf{R}^{n}$ satisfy

$$
|w| \leq d_{\Omega}(x) .
$$

Let $y \in \mathcal{N}_{\partial \Omega_{r_{0}}}(x)$, where $\Omega_{r_{0}}$ is $r_{0^{-}}$neighborhood of $\Omega$. Then by Proposition 9 (c),

$$
\begin{aligned}
|x-y| & =d_{\Omega}(x)+r_{0}, \\
|x+w-y| & \geq d_{\Omega}(x+w)+r_{0}, \\
|x-w-y| & \geq d_{\Omega}(x-w)+r_{0} .
\end{aligned}
$$

Then we have

$$
d_{\Omega}(x+w)-2 d_{\Omega}(x)+d_{\Omega}(x-w) \leq|x+w-y|-2|x-y|+|x-w-y| \leq \frac{C}{r_{0}}|w|^{2},
$$

and so $d_{\Omega}-2 \frac{C}{r_{0}}|x|^{2}$ is concave.

Now we prove Proposition 23. The function $D(x, t)$ defined in (39) is Lipschitz since $\left\{\Omega_{t}\right\}$ is a Lipschitz family of sets. By Proposition 9 the set $\left(\Omega_{t}\right)_{r_{0}}$ satisfies Condition 3 with radius $\frac{r_{0}}{2}$, and

$$
d_{\left(\Omega_{t}\right)_{r_{0}}}^{s}(x)=d_{\Omega_{t}}^{s}(x)+r_{0} \text { for }(x, t) \in E_{r_{0}} .
$$

Then by Lemma 24 the function $D(x, t)-C|x|^{2}$ on the set $E_{r_{0}}$ is concave in $x$ for every $t$. Now by Theorem 1 of Appendix 2 of [Kry87] the function $D(x, t)$ is $(2,1)$ differentiable at $\mathcal{L}^{n+1}$ a.e. point of $E_{r_{0}}$. It follows from Proposition 13 that if $D(\cdot)$ is $(2,1)$ differentiable at a point $\left(x_{0}, t_{0}\right) \in E$, then $\Gamma$ is $(2,1)$ differentiable at $\left(y, t_{0}\right)$, 
where $y \in \mathcal{N}_{\partial \Omega_{t_{0}}}\left(x_{0}\right)$. Note that $x_{0}$ lies in the relative interior of its distance ray if $d_{\Omega_{t_{0}}}$ is twice $x$-differentiable at $x_{0}$. The Proposition is proved.

\section{Properties of MASS TRANSPort DENSity.}

Let $\Omega$ be a bounded set that satisfies the condition 3 with radius $r_{0}$. Let $t>0$. Define a function $a=a_{\Omega, t}: \mathbf{R}^{n} \rightarrow \mathbf{R}^{1}$ as following. Let $x \in \partial \Omega$ be such a point that the surface $\partial \Omega$ is twice differentiable at $x$ and let $\kappa_{1}, \ldots, \kappa_{n-1}$ be the principal curvatures of $\partial \Omega$ at $x$. Introduce the coordinate $s$ on the distance ray $R_{x}$ by $s(z)=$ $|z-x|$ for $z \in R_{x}$. Then $s$ changes in the interval $(0, \gamma(x))$. Define the function $a$ on $R_{x}$ as the following:

$$
a(s)=\frac{1}{t} \prod_{i=1}^{n-1} \frac{1}{1-s \kappa_{i}} \int_{0}^{s} \prod_{i=1}^{n-1}\left(1-\xi \kappa_{i}\right)\left[\frac{\int_{0}^{\gamma(x)} \nu \prod_{i=1}^{n-1}\left(1-\kappa_{i} \nu\right) d \nu}{\int_{0}^{\gamma(x)} \prod_{i=1}^{n-1}\left(1-\kappa_{i} \nu\right) d \nu}-\xi\right] d \xi
$$

By Corollary 22 the function $a$ is now defined a.e. in $\Omega$.

Define $a \equiv 0$ on $\mathbf{R}^{n} \backslash \Omega$. Now $a$ is defined a.e. in $\mathbf{R}^{n}$.

Definition 25. The function $a(\cdot)=a_{\Omega, t}(\cdot)$ is called mass transport density.

Define the function $V: \partial \Omega \rightarrow \mathbf{R}$ at the point of twice differentiability of $\partial \Omega$ as following.

$$
V(x)=\frac{1}{t} \frac{\int_{0}^{\gamma(x)} s \prod_{i=1}^{n-1}\left(1-\kappa_{i} s\right) d s}{\int_{0}^{\gamma(x)} \prod_{i=1}^{n-1}\left(1-\kappa_{i} s\right) d s} .
$$

Proposition 26. Let $\Omega \in \mathbf{R}^{n}$ be a bounded open set that satisfies Condition 3 with radius $r_{0}$. Then there exists a constant $C$ depending only on $r_{0}, n$, diam $\Omega$ such that the following is true. Let $x \in \partial \Omega$ be such point that $\partial \Omega$ be twice differentiable at $x$. Then the mass transport density on the distance ray $R_{x}$, defined by (40) for $s \in[0, \gamma(x)]$, satisfies:

(a) $|a(s)| \leq C$. Derivative $a^{\prime}(s)$ exists and is continuous for $s \in(0, \gamma(x))$.

(b) a(s) satisfies the equation

$$
a^{\prime}(s)-a(s) \sum_{i=1}^{n-1} \frac{\kappa_{i}}{1-\kappa_{i} s}-\frac{s}{t}+V(x)=0
$$

on $(0, \gamma(x))$, the boundary conditions $a(0)=0, a(\gamma(x))=0$, and $a(s)>0$ on $(0, \gamma(x))$.

(c) For $s \in(0, \gamma(x))$ the inequalities hold

$$
|a(s)| \leq C(\gamma(x)-s), \quad|a(s)| \leq C s .
$$

(d) $\left|a^{\prime}(s)\right| \leq C$ on $(0, \gamma(x))$.

Proof. (a) Existence and continuity of $a^{\prime}(s)$ on $(0, \gamma(x))$ are checked explicitly. The bound $|a(s)| \leq C$ will follow from $(c)$. 
(b) The equation is checked explicitly. The boundary conditions follow from $(c)$. The inequality $a(s)>0$ on $(0, \gamma(x))$ holds since either $\kappa_{i}<0$, or $0 \leq \gamma(x) \leq \frac{1}{\kappa_{i}}$ and then, defining

$$
\tilde{V}(s)=\frac{\int_{0}^{s} \nu \prod_{i=1}^{n-1}\left(1-\kappa_{i} \nu\right) d \nu}{\int_{0}^{s} \prod_{i=1}^{n-1}\left(1-\kappa_{i} \nu\right) d \nu}
$$

we calculate:

$$
\begin{aligned}
\frac{d \tilde{V}}{d s} & =\frac{s \prod_{i=1}^{n-1}\left(1-\kappa_{i} s\right) \int_{0}^{s} \prod_{i=1}^{n-1}\left(1-\kappa_{i} \nu\right) d \nu-\prod_{i=1}^{n-1}\left(1-\kappa_{i} s\right) \int_{0}^{s} \nu \prod_{i=1}^{n-1}\left(1-\kappa_{i} \nu\right) d \nu}{\left.\left[\int_{0}^{s} \prod_{i=1}^{n-1}\left(1-\kappa_{i} \nu\right) d \nu\right)\right]^{2}} \\
& =\frac{\prod_{i=1}^{n-1}\left(1-\kappa_{i} s\right)}{\left.\left[\int_{0}^{s} \prod_{i=1}^{n-1}\left(1-\kappa_{i} \nu\right) d \nu\right)\right]^{2}} \int_{0}^{s} \prod_{i=1}^{n-1}\left(1-\kappa_{i} \nu\right)(s-\nu) d \nu>0 \text { for } s \in(0, \gamma(x)),
\end{aligned}
$$

and so

$$
a(s)=\frac{1}{t}[\tilde{V}(\gamma(x))-\tilde{V}(s)] \prod_{i=1}^{n-1} \frac{1}{1-\kappa_{i} s} \int_{0}^{s} \prod_{i=1}^{n-1}\left(1-\kappa_{i} \nu\right) d \nu>0 \text { for } s \in(0, \gamma(x)) .
$$

(c)We write $\gamma$ for $\gamma(x)$ : Denote $\Phi(s)=\int_{0}^{s} \nu \prod_{i=1}^{n-1}\left(1-\kappa_{i} \nu\right) d \nu, \Psi(s)=\int_{0}^{s} \prod_{i=1}^{n-1}(1-$ $\left.\kappa_{i} \nu\right) d \nu$. For $s \in(0, \gamma)$ we calculate:

$$
\begin{aligned}
\operatorname{ta}(s) & =\frac{[\Phi(\gamma) \Psi(s)-\Phi(\gamma) \Psi(\gamma)]+[\Phi(\gamma) \Psi(\gamma)-\Phi(s) \Psi(\gamma)]}{\Psi(\gamma)} \prod_{i=1}^{n-1} \frac{1}{1-\kappa_{i} s} \\
& =(\gamma-s) \frac{-\Phi(\gamma) \Psi^{\prime}\left(s_{1}\right)+\Phi^{\prime}\left(s_{2}\right) \Psi(\gamma)}{\Psi(\gamma)} \prod_{i=1}^{n-1} \frac{1}{1-\kappa_{i} s}
\end{aligned}
$$

where $s_{1}, s_{2} \in[s, \gamma]$. Note, that $\tilde{V}(\xi)=\frac{\Phi(\xi)}{\Psi(\xi)}$. So

$$
\begin{aligned}
t a(s) & =\prod_{i=1}^{n-1} \frac{1}{1-\kappa_{i} s}\left[-\tilde{V}(\gamma) \prod_{i=1}^{n-1}\left(1-\kappa_{i} s_{1}\right)+\prod_{i=1}^{n-1}\left(a-\kappa_{i} s_{2}\right) \cdot s_{2}\right](\gamma-s) \\
& =\left[-\tilde{V}(\gamma) \prod_{i=1}^{n-1} \frac{1-\kappa_{i} s_{1}}{1-\kappa_{i} s}+s_{2} \prod_{i=1}^{n-1} \frac{1-\kappa_{i} s_{2}}{1-\kappa_{i} s}\right](\gamma-s) .
\end{aligned}
$$

If $\kappa_{i} \geq 0$ then for $s^{*} \in(s, \gamma)$ we have $0 \leq s \kappa_{i}<s^{*} \kappa_{i}<1$, and so

$$
0 \leq \frac{1-\kappa_{i} s^{*}}{1-\kappa_{i} s} \leq 1
$$

If $\kappa_{i}<0$ then it follows from the Condition 3 that $\left|\kappa_{i}\right| \leq C$, and so

$$
0 \leq \frac{1-\kappa_{i} s^{*}}{1-\kappa_{i} s}=\frac{1+\left|\kappa_{i}\right| s^{*}}{1+\left|\kappa_{i}\right| s} \leq 1+C \gamma \leq 1+C \operatorname{diam} \Omega
$$

It remains to estimate $\tilde{V}(s)$ for $s \in[0, \gamma]$.

$$
\tilde{V}(s) \leq \frac{\int_{0}^{s} \gamma \prod_{i=1}^{n-1}\left(1-\nu \kappa_{i}\right) d \nu}{\int_{0}^{s} \prod_{i=1}^{n-1}\left(1-\nu \kappa_{i}\right) d \nu}=\gamma \leq \operatorname{diam} \Omega .
$$


So, $t a(s) \leq C$ diam $\Omega(\gamma-s)$, and, since $t>0$,

$$
0<a(s) \leq C(\gamma-s) \text { for } s \in(0, \gamma(x)) .
$$

To estimate $a(s)$ near $s=0$, we compute using (42)

$$
\begin{aligned}
|t a(s)| & =\left|[\tilde{V}(\gamma)-\tilde{V}(s)] \prod_{i=1}^{n-1} \frac{1}{1-\kappa_{i} s} \int_{0}^{s} \prod_{i=1}^{n-1}\left(1-\kappa_{i} \nu\right) d \nu\right| \\
& \leq \gamma\left|\prod_{i=1}^{n-1} \frac{1}{1-\kappa_{i} s} \int_{0}^{s} \prod_{i=1}^{n-1}\left(1-\kappa_{i} \nu\right) d \nu\right|=\gamma s \prod_{i=1}^{n-1} \frac{1-\kappa_{i} s^{*}}{1-\kappa_{i} s}
\end{aligned}
$$

where $s^{*} \in[0, s]$. Since $\kappa_{i} \gamma \leq 1$, then for $s \leq \frac{\gamma}{2}$ we get

$$
0 \leq \frac{1-\kappa_{i} s^{*}}{1-\kappa_{i} s} \leq 2 \text { if } \kappa_{i} \geq 0
$$

and since $\kappa_{i}>-\frac{1}{r}$ we get

$$
0 \leq \frac{1-\kappa_{i} s^{*}}{1-\kappa_{i} s} \leq C(\operatorname{diam}(\Omega), r) \quad \text { if } \kappa_{i}<0 .
$$

So $|\operatorname{ta}(s)| \leq C \gamma s$, and, since $t>0$, we get

$$
|a(s)| \leq C s .
$$

If $s \geq \frac{\gamma}{2}$, then $s \geq \gamma-s$, and from (43)

$$
|a(s)| \leq C(\gamma-s) \leq C s .
$$

(d) We prove that $\left|a^{\prime}(s)\right| \leq C$ for $s \in(0, \gamma)$.

$$
a^{\prime}(s)=a(s) \sum_{i=1}^{n-1} \frac{\kappa_{i}}{1-\kappa_{i} s}+\frac{s}{t}-V(x) .
$$

By (42), $|V(x)| \leq \frac{\gamma}{t}$. It remains to prove that

$$
\left|a(s) \sum_{i=1}^{n-1} \frac{\kappa_{i}}{1-\kappa_{i} s}\right| \leq C
$$

If $\kappa_{i} \geq 0$ then $0 \leq \kappa_{i} \gamma \leq 1$, and since $\gamma>s$ we get:

$$
0 \leq \frac{\kappa_{i}}{1-\kappa_{i} s} \leq \frac{\kappa_{i}}{\kappa_{i} \gamma-\kappa_{i} s}=\frac{1}{\gamma-s}
$$

If $\kappa_{i}<0$, then

$$
\left|\frac{\kappa_{i}}{1-\kappa_{i} s}\right| \leq \frac{1}{s}
$$

Now (44) follows from (c). 


\section{Mass Balance Equation}

Let $\left\{\Omega_{t}\right\}$ be a Lipschitz family of open sets satisfying Condition 3 with radius $r_{0}>0$. Let $\left\{\Omega_{t}\right\}$ satisfy equation (15) on the time-interval $[a, b]$ in the sense described in Theorem 5. Let the function $w(x, t)$ be defined by (13). Let $a(x, t)$ be the function $a_{\Omega_{t}, t}(x)$ from Definition 25.

The purpose of this section is to show that for a.e. $t \in[a, b]$

$$
\int_{\mathbf{R}^{n}} a D w D \phi d z=\int_{\mathbf{R}^{n}}\left(\frac{w}{t}-w_{t}\right) \phi d z
$$

for any $\phi \in C^{\infty}\left(\mathbf{R}^{n}\right)$, where $w_{t}$ denotes $\partial_{t} w$. Thus we show that the function $a(\cdot, t)$ satisfies (18) for a.e $t$.

It follows from Proposition 23 that for a. e. $t$ the following is true: $\mathcal{L}^{n}$ a. e. point $x \in \Omega_{t}$ lies in the relative interior of the distance ray that intersects $\partial \Omega_{t}$ at such point $y \in \partial \Omega_{t}$ that the surface $\Gamma$ is $(2,1)$ differentiable at $(y, t)$, where $\Gamma$ is defined by $(5)$. Fix such $t$. For the rest of this section we drop $t$ in the notation, i.e., we write $\Omega$, $a(x), w(x), w_{t}(x)$ for $\Omega_{t}, a(x, t), w(x, t), w_{t}(x, t)$.

We use same notation $a(\cdot)$ for both mass transport density $a(x)$, a function defined on $\mathbf{R}^{n}$, and for mass transport density on a ray $R_{x}$, the function $a(s)$ defined on $[0, \gamma(x)]$.

Denote by $\tilde{\Gamma}$ the subset of $\partial \Omega$ that consists of all points at which the surface $\Gamma$ is $(2,1)$-differentiable. By choice of $t$ and Proposition 21 we get

$$
\mathcal{H}^{n-1}(\mathcal{B} \backslash \tilde{\Gamma})=0,
$$

where the set $\mathcal{B}$ is defined by (38). From definition of $\mathcal{B}$ it follows that no distance rays of $\Omega$ have their lower ends in the subset $\partial \Omega \backslash \mathcal{B}$ of the boundary. Denote by $R(\tilde{\Gamma})$ the union of all distance rays that have lower ends in $\tilde{\Gamma}$, i.e., $R(\tilde{\Gamma})=\cup_{x \in \tilde{\Gamma}} R_{x}$. Then it follows from Proposition 18 and Remark 20 that

$$
\mathcal{L}^{n}(\Omega \backslash R(\tilde{\Gamma}))=0, \quad \mathcal{L}^{n}\left[G_{k}^{-1}(\Omega \backslash R(\tilde{\Gamma}))\right]=0 \text { for } k=1, \ldots, N .
$$

Let $V(\cdot)$ be the function (41). It follows from (46) that at a. e. point $x$ of $\Omega$ the function $w_{t}(x)$ is given by the expression $\frac{1}{t} V(y)$ where $y \in \mathcal{N}_{\partial \Omega}(x)$. In the next proposition we prove that $w_{t}$ is measurable.

We continue to use local coordinate systems on $\Omega_{r}$, described in Section 3 and notation introduced there, in particular sets $U_{k}$, partition of unity $\Psi_{k}$ and coordinate mappings $G_{k}$ defined by (29), (30) and (27) respectively.

Proposition 27. a) For $k=1, \ldots, n$ there exists a bounded $\mathcal{L}^{n}$ measurable function $v_{k}: U_{k} \rightarrow \mathbf{R}^{1}$ such that

$$
v_{k}\left(x^{\prime}, x_{n}\right)=V\left(G_{k}\left(x^{\prime}, r\right)\right) \quad \text { if } \quad G_{k}\left(x^{\prime}, r\right) \in \tilde{\Gamma}, x_{n} \in\left[r, \tilde{\gamma}_{r, k}\left(x^{\prime}\right)\right] .
$$

b) The function $w_{t}$ is $\mathcal{L}^{n}$ measurable and for any bounded measurable function $\varphi: U_{k} \rightarrow \mathbf{R}^{1}$

$$
\frac{1}{t} \int_{U_{k} \cap\left\{x_{n} \geq r\right\}} v_{k} \varphi J_{n} G_{k} d x=\int_{G_{k}\left(U_{k}\right)} w_{t}\left(\varphi \circ G_{k}^{-1}\right) d x
$$


Proof. a) $\partial \Omega_{r}$ is a $C^{1,1}$ manifold, and so the second fundamental form of $\partial \Omega_{r}$ is defined as a differential form with $L^{\infty}$ coefficients. At all points where $\partial \Omega_{r}$ is twice differentiable the principal curvatures of $\partial \Omega_{r}$ are the roots of the characteristic polynomial of the second fundamental form. To write this in coordinates, we recall that $\partial \Omega_{r} \cap G_{k}\left(U_{k}\right)$ is the graph of a $C^{1,1}$ function $x_{n}=\Phi_{k}\left(x^{\prime}\right)$ defined on the set $\tilde{U}_{k} \in \mathbf{R}^{n-1}$. Then there exist $L^{\infty}$ functions $\left[u_{i j}\left(x^{\prime}\right)\right]_{i, j=1}^{n}$ such that at the points where $D^{2} \Phi_{k}$ exists, $\frac{\partial^{2} \Phi_{k}}{\partial x_{i} \partial x_{j}}=u_{i j}$.

Denote by $P_{x^{\prime}}(s)$ the characteristic polynomial of the matrix $\left[u_{i j}\left(x^{\prime}\right)\right]$ i.e. $P_{x^{\prime}}(s)=$ $\operatorname{det}\left(\left[u_{i j}\left(x^{\prime}\right)\right]-s I_{n-1}\right)$. Note that the coefficients of the polynomial $P_{x^{\prime}}(\cdot)$ are measurable functions of $x^{\prime}$ since they are compositions of polynomials and measurable functions $u_{i j}\left(x^{\prime}\right)$.

The eigenvalues of the matrix $\left[u_{i j}\left(x^{\prime}\right)\right]_{i, j=1}^{n}$ are principal curvatures of $\partial \Omega_{r}$ at the points of twice differentiability of $\Phi_{k}$. Let $x^{\prime}$ be such a point, let $\kappa_{r, 1}, \ldots, \kappa_{r, n-1}$ be principal curvatures of $\partial \Omega_{r}$ in $G_{k}\left(x^{\prime}, 0\right)$, and let $m$ be such number that $\kappa_{r, i} \neq 0$ for $i=1, \ldots, m$ and $\kappa_{r, i}=0$ for $i=m+1, \ldots, n-1$. Then $P_{x^{\prime}}(s)=\prod_{i=1}^{n-1}\left(\kappa_{r, i}-s\right)$, and so

$$
(-1)^{n-1} s^{n-1} P_{x^{\prime}}\left(\frac{1}{s}\right)=\prod_{i=1}^{n-1}\left(1-\kappa_{r, i} s\right)=\prod_{i=1}^{m} \kappa_{r, i} \prod_{i=1}^{m}\left(\frac{1}{\kappa_{r, i}}-s\right) .
$$

For any $x^{\prime} \in U_{k}$ the point $G_{k}\left(x^{\prime}, r\right)$ belongs to $\partial \Omega$ and $\left|G_{k}\left(x^{\prime}, 0\right)-G_{k}\left(x^{\prime}, r\right)\right|=r$. If the surface $\partial \Omega$ is twice differentiable at the point $y=G_{k}\left(x^{\prime}, r\right)$, then, by Proposition 12, the surface $\partial \Omega_{r}$ is twice differentiable at the point $y_{r}=G_{k}\left(x^{\prime}, 0\right)$, and, denoting by $\left\{\kappa_{i}\right\}_{i=1}^{n}$ and $\left\{\kappa_{r, i}\right\}_{i=1}^{n}$ the principal curvatures of $\partial \Omega$ at $y$ and of $\partial \Omega_{r}$ at $y_{r}$, we get

$$
\kappa_{r, i}=\frac{\kappa_{i}}{1+\kappa_{i} r}, \text { so } \frac{1}{\kappa_{r, i}}=\frac{1}{\kappa_{i}}+r \quad\left(\text { if } \kappa_{i} \neq 0\right) .
$$

Then, assuming as before that $\kappa_{1}, \ldots, \kappa_{m} \neq 0$ and $\kappa_{m+1}, \ldots, \kappa_{n-1}=0$, we get if $m \neq 0$

$$
\begin{aligned}
\prod_{i=1}^{n-1}\left(1-\kappa_{i} s\right) & =\prod_{i=1}^{m} \kappa_{i} \prod_{i=1}^{m}\left(\frac{1}{\kappa_{i}}-s\right)=\prod_{i=1}^{m} \kappa_{i} \prod_{i=1}^{m}\left[\frac{1}{\kappa_{r, i}}-(s+r)\right] \\
& =(-1)^{n-1} \frac{\prod_{i=1}^{m} \kappa_{i}}{\prod_{i=1}^{m} \kappa_{r, i}}(s+r)^{n-1} P_{x^{\prime}}\left(\frac{1}{s+r}\right) .
\end{aligned}
$$

If $m=0$ (i.e., all $\left.\kappa_{r, i} \equiv 0\right)$, then $\prod_{i=1}^{n-1}\left(1-\kappa_{i} s\right)=(-1)^{n-1}(s+r)^{n-1} P_{x^{\prime}}\left(\frac{1}{s+r}\right)=1$.

Now we see that at all $x^{\prime}$ as above, the function $V$ defined in (41) can be expressed at the point $G_{k}\left(x^{\prime}, r\right) \in \partial \Omega$ as

$$
V\left(G_{k}\left(x^{\prime}, r\right)\right)=\frac{\int_{0}^{\tilde{\gamma}_{r, k}\left(x^{\prime}\right)-r} s(s+r)^{n-1} P_{x^{\prime}}\left(\frac{1}{s+r}\right) d s}{\int_{0}^{\tilde{\gamma}_{r, k}\left(x^{\prime}\right)-r}(s+r)^{n-1} P_{x^{\prime}}\left(\frac{1}{s+r}\right) d s}
$$

since, by Proposition 9, $\gamma_{\Omega_{r}}(z)=\gamma(z)_{\Omega}+r$ for $z \in \Omega$.

Define a function $\eta=\eta\left(x^{\prime}, x_{n}\right)$ on $\tilde{U}_{k} \times \mathbf{R}^{1}$ by the right-hand side of (49). This function $\eta$ is $\mathcal{L}^{n}$-measurable: $\eta$ does not depend on $x_{n}$, the coefficients of the polynomial $P_{x^{\prime}}(\cdot)$ are measurable functions of $x^{\prime}$ and by Proposition 15, the functions $\tilde{\gamma}_{r, k}$ are measurable functions of $x^{\prime}$. 
Then it follows from Proposition 15 that the function

$$
v_{k}\left(x^{\prime}, x_{n}\right)=\eta\left(x^{\prime}, x_{n}\right) \chi_{U_{k}}\left(x^{\prime}, x_{n}\right)
$$

satisfies all properties asserted in (a) (note that the right-hand side of (47) is bounded by $\operatorname{diam} \Omega$ ).

By Lemma 16 the function $v_{k} \circ G_{k}^{-1}$ is $\mathcal{L}^{n}$-measurable. By (46) we get

$$
\begin{aligned}
& \frac{1}{t} v_{k} \circ G_{k}^{-1}=w_{t} \quad \text { at } \mathcal{L}^{n} \text { a.e. point of } G_{k}\left(U_{k} \cap\left\{x_{n} \geq r\right\}\right), \\
& \text { and } \quad w_{t} \equiv 0 \quad \text { on } \quad G_{k}\left(U_{k} \cap\left\{x_{n}<r\right\}\right)
\end{aligned}
$$

and so $w_{t}$ is measurable.

Since map $G_{k}$ is one-to-one and Lipschitz on $U_{k}$, and since $U_{k}$ is measurable, we get by Theorem 3.2.5 of [Fed69] and Lemma 16 that for every bounded measurable function $\varphi$

$$
\int_{U_{k}} v_{k} \varphi J_{n} G_{k} d z=\int_{G_{k}\left(U_{k}\right)}\left(v_{k} \circ G_{k}^{-1}\right)(x)\left(\varphi \circ G_{k}^{-1}\right)(x) d x
$$

Now (48) follows from (51), (50).

Corollary 28. The function $\frac{w}{t}-w_{t}$ is measurable, and for each $\varphi \in C^{\infty}\left(\mathbf{R}^{n}\right)$

$$
\int_{\mathbf{R}^{n}}\left(\frac{w}{t}-w_{t}\right) \varphi d z=\frac{1}{t} \sum_{k=1}^{N} \int_{G_{k}^{-1}(R(\tilde{\Gamma}))}\left(w \circ G_{k}-v_{k}\right)\left(\varphi \circ G_{k}\right)\left(\Psi_{k} \circ G_{k}\right) J_{n} G_{k} d x .
$$

Proof. We have

$$
\frac{w}{t}-w_{t}=\sum_{k=1}^{N}\left(\frac{w}{t}-w_{t}\right) \Psi_{k}
$$

Since supp $\Psi_{k} \subset G_{k}\left(U_{k}\right)$, the integrand on the right-hand side of (52) is measurable by Propositions 27 and 18. It is also clear that the integrand on the right-hand side is bounded.

Then from Proposition 27 (b) and area formula, we get

$$
\int_{\mathbf{R}^{n}}\left(\frac{w}{t}-w_{t}\right) \varphi d z=\frac{1}{t} \sum_{k=1}^{N} \int_{\mathbf{R}^{n}}\left(w \circ G_{k}-v_{k}\right)\left(\varphi \circ G_{k}\right)\left(\Psi_{k} \circ G_{k}\right) J_{n} G_{k} d x .
$$

This formula is true for each $\varphi \in C^{\infty}$, and, by approximation, for every bounded measurable $\varphi$. Now (46) implies (52).

Now we prove that the mass transport density $a(\cdot)$ is measurable. The proof is similar to the proof of Proposition 27.

Proposition 29. The function $a(\cdot)$ is $\mathcal{L}^{n}$-measurable function on $\mathbf{R}^{n}$. The function $a \circ G_{k}^{-1}$ is $\mathcal{L}^{n}$-measurable function on $U_{k}$. 
Proof. For $\left(x^{\prime}, s\right)$ such that

$$
G_{k}\left(x^{\prime}, r\right) \in \tilde{\Gamma}, \quad s \in\left[r, \tilde{\gamma}_{r, k}\left(x^{\prime}, r\right)\right],
$$

the function $a \circ G_{k}\left(x^{\prime}, s\right)$ is given by expression (40), where the curvatures are computed at the point $G_{k}\left(x^{\prime}, r\right)$. Calculations similar to those in Proposition 27 show that this expression can be rewritten using the characteristic polynomial $P_{x^{\prime}}(\cdot)$ of the matrix $D^{2} \Phi_{k}\left(x^{\prime}\right)$ as following:

$$
\begin{gathered}
a \circ G_{k}\left(x^{\prime}, s\right)=\frac{1}{t} \frac{\int_{0}^{s-r}(\nu+r)^{n-1} P_{x^{\prime}}\left(\frac{1}{\nu+r}\right) d \nu}{(s+r)^{n-1} P_{x^{\prime}}\left(\frac{1}{s+r}\right)} \times \\
{\left[\frac{\int_{0}^{\tilde{\gamma}_{k, r}\left(x^{\prime}\right)-r} \nu(\nu+r)^{n-1} P_{x^{\prime}}\left(\frac{1}{\nu+r}\right) d \nu}{\int_{0}^{\tilde{\gamma}_{k, r}\left(x^{\prime}\right)-r}(\nu+r)^{n-1} P_{x^{\prime}}\left(\frac{1}{\nu+r}\right) d \nu}-\frac{\int_{0}^{s-r} \nu(\nu+r)^{n-1} P_{x^{\prime}}\left(\frac{1}{\nu+r}\right) d \nu}{\int_{0}^{s-r}(\nu+r)^{n-1} P_{x^{\prime}}\left(\frac{1}{\nu+r}\right) d \nu}\right]}
\end{gathered}
$$

By (46), the function $a \circ G_{k}^{-1}(\cdot)$ is defined by the above formula at a.e. point of $U_{k}$, and is $\mathcal{L}^{n}$-measurable function of variables $x^{\prime}, s$ on $U_{k}$ since it is a rational function of variables $s$ and $\tilde{\gamma}_{k, r}\left(x^{\prime}\right)$ with measurable coefficients and $\tilde{\gamma}_{k, r}\left(x^{\prime}\right)$ is $\mathcal{L}^{n-1}$ measurable function of $x^{\prime}$. By Lemma 16, the function $a(\cdot)$ is measurable.

Proof of (45). We will transform the right-hand side of (52). From (30) we get

$$
\Psi_{k}\left(G_{k}\left(x^{\prime}, s\right)\right)=\Psi_{k}\left(G_{k}\left(x^{\prime}, r\right)\right), \quad s \in\left(r, \tilde{\gamma}_{r, k}\left(x^{\prime}\right)\right) .
$$

Let $\tilde{G}_{k}: \tilde{U}_{k} \rightarrow \partial \Omega$ be the map defined by

$$
\tilde{G}_{k}\left(x^{\prime}\right)=G_{k}\left(x^{\prime}, r\right) .
$$

Then for $k=1, \ldots, N$, using definitions of $w, v_{k}, \Psi_{k}$ we compute

$$
\begin{aligned}
& \frac{1}{t} \int_{G_{k}^{-1}(R(\tilde{\Gamma}))}\left(w \circ G_{k}\right.\left.-v_{k}\right)\left(\varphi \circ G_{k}\right)\left(\Psi_{k} \circ G_{k}\right) J_{n} G_{k} d x \\
&=\int_{\tilde{G}_{k}^{-1}(\tilde{\Gamma})} \Psi_{k}\left(G_{k}\left(x^{\prime}, r\right)\right)\left\{\int_{r}^{\tilde{\gamma}_{r, k}\left(x^{\prime}\right)} \frac{1}{t}[s-r\right. \\
&\left.\left.\quad-v_{k}\left(x^{\prime}, s\right)\right] \varphi\left(G_{k}\left(x^{\prime}, s\right)\right) J_{n} G_{k}\left(x^{\prime}, s\right) d s\right\} d x^{\prime}
\end{aligned}
$$

Let $x^{\prime} \in \mathbf{R}^{n-1}$ be such that $G_{k}\left(x^{\prime}, r\right) \in \tilde{\Gamma}$. Then $\partial \Omega$ is twice differentiable at $G_{k}\left(x^{\prime}, r\right)$, and so

$$
\frac{1}{t} v_{k}\left(x^{\prime}, s\right)=V\left(G_{k}\left(x^{\prime}, r\right)\right) \quad \text { for } s \in\left[r, \tilde{\gamma}_{r, k}\left(x^{\prime}\right)\right] .
$$

By Proposition 12, $\partial \Omega_{r}$ is twice differentiable at $G_{k}\left(x^{\prime}, 0\right)$. Let $\kappa_{1}, \ldots, \kappa_{n-1}$ be principal curvatures of $\partial \Omega$ at $G_{k}\left(x^{\prime}, r\right)$, and $\kappa_{r, 1}, \ldots, \kappa_{r, n-1}$ be principal curvatures of $\partial \Omega_{r}$ at $G_{k}\left(x^{\prime}, 0\right)$. Then $\kappa_{r, i}=\frac{\kappa_{i}}{1+r \kappa_{i}}$. Let $y=G_{k}\left(x^{\prime}, r\right)$. Then the mass transport density on 
the ray $R_{y}$ satisfies ODE from Proposition 26(b). Thus we get for $s \in\left[r, \tilde{\gamma}_{r, k}\left(x^{\prime}\right)\right]=$ $\left[r, \gamma_{\Omega}(y)+r\right]:$

$$
\begin{aligned}
\frac{1}{t}\left(s-r-v_{k}\right) & =-\frac{d a(s-r)}{d s}+a(s-r) \sum_{i=1}^{n-1} \frac{\kappa_{i}}{1-\kappa_{i}(s-r)} \\
& =-\frac{d a(s-r)}{d s}+a(s-r) \sum_{i=1}^{n-1} \frac{\kappa_{r, i}}{1-\kappa_{r, i} s}
\end{aligned}
$$

where $a(s)$ is mass transport density on the ray $R_{y}$.

By Proposition 26, $\frac{d a}{d s}$ exists and is continuous and bounded on the interval $\left(0, \tilde{\gamma}_{r, k}\left(x^{\prime}\right)-\right.$ $r)$, and

$$
a(0)=a\left(\tilde{\gamma}_{r, k}\left(x^{\prime}\right)-r\right)=0
$$

We also see by (28) that

$$
\frac{\partial J_{n} G_{k}}{\partial s}\left(x^{\prime}, s\right)=J_{n} G_{k}\left(x^{\prime}, s\right) \sum_{i=1}^{n-1} \frac{-\kappa_{r, i}}{1-\kappa_{r, i} s} .
$$

Then we calculate, integrating by parts:

$$
\begin{gathered}
\int_{r}^{\tilde{\gamma}_{r, k}\left(x^{\prime}\right)} \frac{1}{t}\left[s-r-v_{k}\left(x^{\prime}, s\right)\right] \varphi\left(G_{k}\left(x^{\prime}, s\right)\right) J_{n} G_{k}\left(x^{\prime}, s\right) d s= \\
\int_{r}^{\tilde{\gamma}_{r, k}\left(x^{\prime}\right)}\left[-\frac{d a(s-r)}{d s}-a(s-r) \frac{\frac{\partial}{\partial s} J_{n} G_{k}\left(x^{\prime}, s\right)}{J_{n} G_{k}\left(x^{\prime}, s\right)}\right] \varphi\left(G_{k}\left(x^{\prime}, s\right)\right) J_{n} G_{k}\left(x^{\prime}, s\right) d s \\
=\int_{r}^{\tilde{\gamma}_{r, k}\left(x^{\prime}\right)} a(s-r) \frac{\partial}{\partial s} \varphi\left(G_{k}\left(x^{\prime}, s\right)\right) J_{n} G_{k}\left(x^{\prime}, s\right) d s
\end{gathered}
$$

This allows to derive from (52), (55) and (54) the following equality:

$$
\begin{aligned}
& \quad \int_{\mathbf{R}^{n}}\left(\frac{w}{t}-w_{t}\right) \varphi d z= \\
& \sum_{k=1}^{N} \int_{\tilde{G}_{k}^{-1}(\tilde{\Gamma})} \int_{r}^{\tilde{\gamma}_{r, k}\left(x^{\prime}\right)} \Psi_{k}\left(G_{k}\left(x^{\prime}, s\right)\right) a\left(G_{k}\left(x^{\prime}, s\right)\right) \frac{\partial}{\partial s} \varphi\left(G_{k}\left(x^{\prime}, s\right) J_{n} G_{k}\left(x^{\prime}, s\right) d s d x^{\prime}\right.
\end{aligned}
$$

Let $z=G_{k}\left(x^{\prime}, s\right)$. Using the fact that distance function is twice differentiable on $R(\tilde{\Gamma})$, we get:

$$
\begin{aligned}
\frac{\partial}{\partial s}\left(\varphi\left(G_{k}\left(x^{\prime}, s\right)\right)\right. & =(D \varphi)\left(G_{k}\left(x^{\prime}, s\right)\right) \cdot \frac{\partial}{\partial s}\left(G_{k}\left(x^{\prime}, s\right)\right) \\
& =D \varphi(z) \cdot D d_{\Omega}(z) .
\end{aligned}
$$

We insert the right-hand side of (58) into the right-hand side of (57) and use area formula and (46) to change variables from $\left(x^{\prime}, s\right)$ to $z$ in the integral on the righthand side of (57). Then using that $\sum_{k} \Psi_{k} \equiv 1$ on $\Omega^{0} \backslash \mathcal{R}$ and that $a \equiv 0$ on $\mathcal{R}$ and in $\mathbf{R}^{n} \backslash \Omega$, we get (45). 


\section{Proof of Theorem 5}

To conclude the proof of Theorem 5 we need to demonstrate the following. Let, as above, $w(x)=d_{\Omega}(x)$, and let $v \in W^{1, \infty}\left(\mathbf{R}^{n}\right)$ satisfy $|D v| \leq 1$ a.e. Then

$$
\int_{\mathbf{R}^{n}}\left(\frac{w}{t}-w_{t}\right) w \geq \int_{\mathbf{R}^{n}}\left(\frac{w}{t}-w_{t}\right) v .
$$

We show that this follows from (45). By approximation, (45) is true for Lipschitz $\varphi$. Let $\varphi=w-v$. Then by (45) that it is enough to prove that

$$
\int_{\mathbf{R}^{n}} a D w(D w-D v) \geq 0 .
$$

But, since $a \geq 0$ in $\mathbf{R}^{n}$ and $a \equiv 0$ outside $\Omega^{0}$ and on $\mathcal{R}$, and $|D w| \equiv 1$ on $\Omega \backslash \mathcal{R}$,

$$
\int_{\mathbf{R}^{n}} a D w D w=\int_{\mathbf{R}^{n}} a \geq \int_{\mathbf{R}^{n}} a D w D v
$$

since $|D w D v| \leq \sqrt{|D w|^{2}|D v|^{2}} \leq 1$.

Theorem 5 is proved.

\section{Proof of Theorem 6}

The proof of Theorem 6 is similar to the proof Theorem 5. We give a formal calculation. Each step can be justified the same way as in the proof of Theorem 5 .

Fix $t \in[a, b]$. Define

$$
\begin{aligned}
& \mathcal{D}_{1}=\mathcal{D}_{1}(t)=\left\{x \in \Omega_{t}^{1} \mid d_{\Omega_{t}^{1}}(x)>d_{\Omega_{t}^{2}}(x)\right\}, \\
& \mathcal{D}_{2}=\mathcal{D}_{2}(t)=\left\{x \in \Omega_{t}^{2} \mid d_{\Omega_{t}^{2}}(x)>d_{\Omega_{t}^{1}}(x)\right\} .
\end{aligned}
$$

Let $r>0$, let $\Omega_{r}^{1}=\Omega_{t, r}^{1}$ and $\Omega_{r}^{2}=\Omega_{t, r}^{2}$ be $r$-neighborhoods of $\Omega^{1}=\Omega_{t}^{1}$ and $\Omega^{2}=\Omega_{t}^{2}$, and let $r$ be so small that $\partial \Omega_{r}^{1}$ and $\partial \Omega_{r}^{2}$ are $C^{1,1}$ manifolds.

Let $l=1,2$. Let $\left\{\tilde{U}_{k}^{l}\right\}_{k=1}^{N_{l}}$ and $\left\{U_{k}^{l}\right\}_{k=1}^{N_{l}}$ be the coordinate neighborhoods associated the set $\Omega_{r}^{l}$ same way as $\left\{\tilde{U}_{k}\right\},\left\{U_{k}\right\}$ with $\Omega_{r}$ in the proof of Theorem 5. Let $G_{k}^{l}$ :

$U_{k}^{l} \rightarrow \mathbf{R}^{n}$ be the corresponding coordinate mappings (27) and let $\left\{\Psi_{k}^{l}\right\}_{k=0}^{N_{l}+1}$ be the corresponding partitions of unity (30). Let $\tilde{\gamma}_{r, k}^{l}$ be the functions defined as in (31) from the functions $\gamma_{\Omega_{r}^{l}}$ using the coordinate mappings $G_{k}^{l}$ for $k=1, \ldots, N_{l}$. Define the following functions $\tilde{\delta}_{r, k}^{l}$ for $k=1, \ldots, N_{l}$. Let $\delta(\cdot) \equiv \gamma_{\Omega^{1} \cap \Omega^{2}}(\cdot)$. Then

$$
\tilde{\delta}_{r, k}^{l}\left(x^{\prime}\right)= \begin{cases}\delta\left(G_{k}^{l}\left(x^{\prime}, r\right)\right)+r, & \text { if } x^{\prime} \in \operatorname{cl}\left(\tilde{U}_{k}^{l}\right) \quad \text { and } \quad G_{k}^{l}\left(x^{\prime}, r\right) \in \partial\left(\Omega^{1} \cap \Omega^{2}\right) ; \\ r, & \text { otherwise. }\end{cases}
$$

Note that

$$
\left(G_{k}^{l}\right)^{-1}\left(\mathcal{D}_{l}\right) \cap U_{k}^{l}=\left\{\left(x^{\prime}, s\right) \quad \mid x^{\prime} \in \tilde{U}_{k}^{l}, s \in\left[\tilde{\delta}_{r, k}^{l}\left(x^{\prime}\right), \tilde{\gamma}_{r, k}^{l}\left(x^{\prime}\right)\right]\right\} .
$$

The functions $\tilde{\delta}_{r, k}^{l}$ are uppersemicontinuous, the proof is similar to the proof of Proposition [15, using the properties of sets satisfying Condition 5 . 
Define the mass transport density $a(\cdot)$. Let $a \equiv 0$ on $\mathbf{R}^{n} \backslash\left(\Omega^{1} \cup \Omega^{2}\right)$. To define $a$ in $\Omega^{1} \cup \Omega^{2}$ it is enough to define $a$ on $\mathcal{D}_{1} \cup \mathcal{D}_{2}$. Let $x \in \mathcal{D}_{l} \backslash \mathcal{R}_{l}$, where $l=1,2$. Let $y \in \mathcal{N}_{\partial \Omega_{l}}(x)$ and $s=|x-y|$. Then we define

$$
a(x)=\frac{1}{t} \prod_{i=1}^{n-1} \frac{1}{1-s \kappa_{i}} \int_{\delta(y)}^{s} \prod_{j=1}^{n-1}\left(1-\kappa_{j} \xi\right)\left[\frac{\int_{\delta(y)}^{\gamma^{l}(y)} \nu \prod_{k=1}^{n-1}\left(1-\kappa_{k} \nu\right) d \nu}{\int_{\delta(y)}^{\gamma^{l}(y)} \prod_{k=1}^{n-1}\left(1-\kappa_{k} \nu\right) d \nu}-\xi\right] d \xi
$$

where $\kappa_{k}$ are curvatures of $\partial \Omega_{t}^{l}$ at $y$. Then $a$ satisfies the equation (20) on subintervals of distance rays of $\Omega_{t}^{l}$ that lie in $\mathcal{D}_{l}$, and $a \equiv 0$ on $\partial \mathcal{D}_{l}$ and on $\mathcal{R}_{l}, l=1,2$. We calculate, denoting by $\kappa_{r, i}$ the principal curvatures of $\partial \Omega_{r}^{l}$ at the point $y_{r} \in \Omega_{r}^{l}$ nearest to $x$

$$
\begin{aligned}
\int_{\mathbf{R}^{n}}\left(\frac{w}{t}-\right. & \left.w_{t}\right) \varphi d z \\
= & \frac{1}{t} \sum_{l=1}^{2} \sum_{k=1}^{N_{l}} \int_{U_{k}^{l} \cap\left(G_{k}^{l}\right)^{-1}\left(\mathcal{D}_{l}\right)}\left(w \circ G_{k}^{l}-V \circ G_{k}^{l}\right)\left(\varphi \circ G_{k}^{l}\right)\left(\Psi_{k}^{l} \circ G_{k}^{l}\right) J_{n} G_{k}^{l} d x \\
= & \sum_{l=1}^{2} \sum_{k=1}^{N_{l}} \int_{\tilde{U}_{k}^{l}} \Psi_{k}^{l}\left(G_{k}^{l}\left(x^{\prime}, 0\right)\right) \\
& \times \int_{\tilde{\delta}_{r, k}^{l}\left(x^{\prime}\right)}^{\tilde{\gamma}_{r, k}^{l}\left(x^{\prime}\right)} \frac{1}{t}\left[(s-r)-V\left(G_{k}^{l}\left(x^{\prime}, 0\right)\right)\right]\left(\varphi\left(G_{k}^{l}\left(x^{\prime}, s\right)\right) J_{n} G_{k}^{l}\left(x^{\prime}, s\right) d s d x^{\prime}\right. \\
= & \sum_{l=1}^{2} \sum_{k=1}^{N_{l}} \int_{\tilde{U}_{k}^{l}} \Psi_{k}^{l}\left(G_{k}^{l}\left(x^{\prime}, 0\right)\right) \int_{\tilde{\delta}_{r, k}^{l}\left(x^{\prime}\right)}^{l}\left(-\frac{d a\left(G_{k}^{l}\left(x^{\prime}, s\right)\right)}{d s}\right. \\
& \left.+a\left(G_{k}^{l}\left(x^{\prime}, s\right)\right) \sum_{i=1}^{n-1} \frac{\kappa_{r, i}}{1-\kappa_{r, i} s}\right) \varphi J_{n} G_{k}^{l}\left(x^{\prime}, s\right) d s d x^{\prime} \\
= & \sum_{l=1}^{2} \sum_{k=1}^{N_{l}} \int_{\tilde{U}_{k}^{l}} \Psi_{k}^{l}\left(G_{k}^{l}\left(x^{\prime}, 0\right)\right) \\
& \times \int_{\tilde{\delta}_{r, k}^{l}\left(x^{\prime}\right)}^{\tilde{\gamma}_{r, k}^{l}\left(x^{\prime}\right)} a\left(G_{k}^{l}\left(x^{\prime}, s\right)\right) \frac{\partial \varphi\left(G_{k}^{l}\left(x^{\prime}, s\right)\right)}{\partial s} J G_{k}^{l}\left(x^{\prime}, s\right) d s d x^{\prime} \\
= & \sum_{\mathbf{R}^{n}}^{2} \sum_{k=1}^{N_{l}} \int_{G_{k}^{l}\left(U_{k}^{l}\right) \cap \mathcal{D}_{l}} a(z) \Psi_{k}^{l}(z) D \varphi(z) D w(z) d z \\
& \sum_{i} D(z .
\end{aligned}
$$

Thus we have proved that the mass balance equation is satisfied. This implies Theorem 6 . 


\section{Compression MOLDing MODEL}

Compression molding is the process of deformation of an incompressible plastic material between two horizontal plates. The following simplified mathematical model of the process was derived by G. Aronsson [Ar95] based on Hele-Show model for nonNewtonian fluid. Suppose that the distance between the horizontal plates is small. Then we can assume that the region occupied by plastic at each time $t$ has the form $U_{t}=\Omega_{t} \times\left[0, h_{t}\right]$ where $\Omega_{t} \subset \mathbf{R}^{2}$, and that the pressure in plastic does not depend on the vertical coordinate, i.e., pressure is the function $u(x, t)$ where $x \in \Omega_{t}$. Evolution of rescaled $\Omega_{t}$ and $u(x, t)$ is described by the following free boundary problem. Given an open set $\Omega_{0} \in \mathbf{R}^{2}$ find an expanding family of open sets $\Omega_{t} \in \mathbf{R}^{2}, t \geq 0$, and a function $u(x, t)$ defined on $\cup_{t}\left(\Omega_{t} \times\{t\}\right)$ such that

$$
\begin{aligned}
-\operatorname{div}\left(|D u|^{p-2} D u\right)=1 & \text { in } \Omega_{t}, \\
u=0 & \text { on } \Gamma_{t}, \\
V=|D u|^{p-2} & \text { on } \Gamma_{t},
\end{aligned}
$$

where $\Gamma_{t}=\partial \Omega_{t}, V$ denotes the outer normal velocity of $\Gamma_{t}$. Condition (62) means that the free boundary $\Gamma_{t}$ moves with the velocity of the flow.

In the paper [AE] the asymptotic limit as $p \rightarrow \infty$ in the problem (60) - 62 was considered. This limit corresponds to the case of highly non-Newtonian fluid.

It was shown in [AE that formally sending to a limit in (60) - (62) one obtains the following problem. Find a family $\left\{\Omega_{t}\right\}$ of open subsets of $\mathbf{R}^{2}$, a function $u(x, t)$, and a mass transport density function $a(x, t)$ satisfying:

$$
\begin{cases}|D u| \leq 1 & \text { a.e. in } \Omega_{t}, \\ a \geq 0 & \text { a.e. in } \Omega_{t}, \\ \operatorname{supp}(a) \in\{|D u|=1\}, & \\ -\operatorname{div}(a D u)=1 & \text { in } \Omega_{t},\end{cases}
$$

where the last equation is understood in the weak sense, and

$$
u=0, \quad V=a \text { on } \Gamma_{t},
$$

where $V$ is the outer normal velocity of $\Gamma_{t}$.

It was shown in $\mathrm{AE}$ that solutions of (63), (64) have the form

$$
u(x, t)=d_{\Omega_{t}}(x),
$$

where the right-hand side is defined by (1), and that formally the problem (63) - (64) can be rewritten as following. Find $\left\{\Omega_{t}\right\}$ such that

$$
w-\partial_{t} w \in \partial I_{\infty}[u]
$$

where

$$
w(\cdot, t)=\chi_{\Omega_{t}}(\cdot),
$$

where $\chi_{\Omega_{t}}(\cdot)$ is the indicator function of $\Omega_{t}$ that equals 1 inside and 0 outside $\Omega_{t}$. Existence of a weak solution of (65) - (66) was proved in [AE]. Namely, there exists a 
family $\left\{\Omega_{t}\right\}$ of sets of finite perimeter such that $\partial_{t} w$ is a nonnegative Radon measure and

$$
\int_{\mathbf{R}^{2}} w(x, t)(v(x)-u(x, t)) d x \leq \int_{\mathbf{R}^{2}}(v-u(\cdot, t)) d\left(\partial_{t} w(\cdot, t)\right)
$$

$$
\text { for every } v \text { with }|D v| \leq 1 \text {, for a.e. } t \text {. }
$$

The following law of motion of the free boundary $\Gamma_{t}$ was derived in AE by a formal calculation:

$$
V=\gamma\left(1-\frac{\kappa \gamma}{2}\right)
$$

where

$$
\left\{\begin{array}{l}
V=\text { outer normal velocity of } \Gamma_{t} \\
\gamma=\text { function } \gamma_{\Omega_{t}}(\cdot) \text { defined by (21) } \\
\kappa=\text { curvature of } \Gamma_{t}
\end{array}\right.
$$

The equation (69) was derived as following. Starting from (63), we perform calculations similar to the ones that lead from (18) to (20). Thus we deduce that equation (63) can be formally rewritten on each distance ray $R_{x}$, where $x \in \Gamma_{t}$, as ODE

$$
a^{\prime}(s)-a(s) \frac{\kappa}{1-\kappa s}+1=0, \quad s \in\left(0, \gamma_{\Omega_{t}}(x)\right) .
$$

By the nature of mass transfer process in the compression molding model (i.e., mass transfer from within the set onto the boundary), we expect that the mass transport density equals to zero on the ridge of $\Omega_{t}$ and equals to the outer normal velocity at the boundary. This translates into the following boundary conditions for the ODE (70) on $R_{x}$ :

$$
a(0)=V(x), \quad a(\gamma(x))=0 .
$$

The function $a(\cdot)$ and the constant $V$ can be found from (70), (71). $V$ has the expression (69).

In this section we prove the connection between the variational equation (66) and the geometric equation (69).

Let $E, \Gamma$ be defined by (5).

Theorem 30. Let $\left\{\Omega_{t}\right\}, t \in \mathbf{R}_{+}^{1}$, be an expanding, locally Lipschitz continuous family of open bounded sets. Let for every $t \in \mathbf{R}_{+}^{1}$ the set $\Omega_{t}$ satisfy Condition 3 with radius $r_{0}>0$. Suppose that the equation (69) is satisfied at every point of $(2,1)$ differentiability of the surface $\Gamma$. Then the equation (60) is satisfied for a.e. $t \in \mathbf{R}^{1}$, where the functions $u(x, t), w(x, t)$ are defined by (65), (6x).

Proof. The proof goes along the lines of the proof of Theorem 5. We will sketch the proof and present some details for the parts that are different from the proof of Theorem 5 .

The main steps of the proof are following:

Step 1. Definition and properties of mass transport density.

Step 2. Show that the main mass balance equation is satisfied. 
Step 3. Show that the assertion of the Theorem follows from the main mass balance equation.

We discuss each step.

Step 1.

Let $(x, t)$ be a point of $(2,1)$ differentiability of the surface $\Gamma$ in $\mathbf{R}^{2} \times \mathbf{R}^{1}$. Define mass transport density $a(y, t)=a(s)$ on the distance ray $R_{x}$ of $\Omega_{t}$ as the solution of (70), (71). Do this for every such point $(x, t)$. It follows from the hypothesis of the Theorem and from Proposition 23 that $a(y, t)$ is now defined at $\mathcal{L}^{3}$ a.e. point of the set $E=\cup_{t}\left(\Omega_{t} \times\{t\}\right) \subset \mathbf{R}^{2} \times \mathbf{R}_{+}^{1}$. Define $a(y, t)$ by zero at all other points of $\mathbf{R}^{2} \times \mathbf{R}_{+}^{1}$.

Solving (70), (71) (and taking into account (69)) we see that on the ray $R_{x}$

$$
a(s)=\frac{1-\kappa s}{2 \kappa}-\frac{(1-\kappa \gamma)^{2}}{2 \kappa(1-\kappa s)} .
$$

This function satisfies assertions (a) and (d) of Proposition 26. Instead of assertion (c) of Proposition 26 we have

$$
|a(s)| \leq C(\gamma(x)-s), \quad|a(s)-V(x)| \leq C s .
$$

To see this, we note that from the conditions of the Theorem and (69) it follows that

$$
V(x) \leq C\left(r_{0}, \operatorname{diam} \Omega_{t}\right) .
$$

Then the assertions (a) and (d) of Proposition 26 for the function $a(s)$ defined by (72) follow from (73) like in the proof of Proposition 26.

Let us prove (73). We can rewrite $(72)$ as

$$
a(s)=\frac{\gamma-s}{2}\left(1+\frac{1-\kappa \gamma}{1-\kappa s}\right) .
$$

Now, since $0 \leq s \leq \gamma \leq \operatorname{diam} \Omega_{t}$ and $\kappa \gamma \leq 1$ and $\kappa \geq-\frac{1}{r_{0}}$, we get the estimate

$$
0 \leq \frac{1-\kappa \gamma}{1-\kappa s} \leq C\left(r_{0}, \operatorname{diam} \Omega_{t}\right)
$$

and the first inequality of (73) follows. To prove the second, we calculate using (71)

$$
a(s)-V=a(s)-a(0)=\frac{s}{2}\left[-1-\frac{(1-\kappa \gamma)^{2}}{1-\kappa s}\right],
$$

and use (74) and the inequality

$$
0 \leq 1-\kappa \gamma \leq C\left(r_{0}, \operatorname{diam} \Omega_{t}\right)
$$

to finish the proof of $(73)$.

Step 2.

The purpose of this step is to prove that for any smooth function $\varphi \in C_{c}^{1}\left(\mathbf{R}^{2} \times\right.$ $(0, T))$ we have

$$
\int_{0}^{T} \int_{\mathbf{R}^{2}} w\left(\varphi+\varphi_{t}\right)-a D u D \varphi=0 .
$$

This is main mass balance equation for compression molding model. 
The proof follows Section 5 .

Fix $t$. We use local coordinate systems on $\left(\Omega_{t}\right)_{r}$ where $0<r \leq r_{0}$, defined in Section 3 and notation introduced there, in particular sets $\tilde{U}_{k}, U_{k}$, partition of unity $\Psi_{k}$, coordinate mappings $G_{k}$ and functions $\tilde{\gamma}_{r, k}$ defined by (29), (30), (27) and (31) respectively $(\mathrm{k}=1, \ldots, \mathrm{N})$.

We use notation $E_{r}, \Gamma_{r}$ introduced in (26). From Proposition 9 it follows that for $r \in\left[0, r_{0}\right]$

$$
\Gamma_{r}=\left\{(x, t) \in \mathbf{R}^{2} \times(0, T) \mid d_{\Omega_{t}}^{s}(x)=-r\right\}
$$

The function

$$
(x, t) \rightarrow d_{\Omega_{t}}^{s}(x)
$$

is Lipschitz since $\left\{\Omega_{t}\right\}$ is a Lipschitz family of sets. Then by Proposition 3.2.15 of Fed69, for a.e. $r \in\left[0, r_{0}\right]$

$$
\text { set } \Gamma_{r} \text { is }\left(\mathcal{H}^{2}, 2\right) \text { rectifiable. }
$$

In addition, by Lemma 24, the function $(x, t) \rightarrow d_{\Omega_{t}}^{s}(x)-C|x|^{2}$, where $C$ is large enough depending on $r_{0}$, is concave in $x$ for every $t$ in the set $E_{r_{0}}$. Then it follows from Theorem 1 of Appendix 2 of [Kry87 and Propositions 23(b) and 11 that for a.e. $r \in\left[0, r_{0}\right]$

$$
\text { surface } \Gamma_{r} \text { is }(2,1) \text { differentiable at } \mathcal{H}^{2} \text { a.e. point; }
$$

functions $\partial_{t} d_{\Omega_{t}}^{s}(x), D d_{\Omega_{t}}^{s}(x), D^{2} d_{\Omega_{t}}^{s}(x)$ are bounded and $\mathcal{H}^{2}$ measurable on $\Gamma_{r}$.

Function $(x, t) \rightarrow \gamma_{\Omega_{t}}(x)$ is uppersemicontinuous since the family of sets $\left\{\Omega_{t}\right\}$ is Lipschitz. Then similar to Propositions 27, 29 and Corollary 28 we prove that the mass transport density $a(y, t)$ defined at Step 1 is Lebesgue measurable in $\mathbf{R}^{2} \times \mathbf{R}_{+}^{1}$. In the proof we use (78) (i.e., we choose such $r$ in the definition of local coordinate systems that (78) is satisfied).

It follows from Proposition 23 that for a.e. $t$ we have the following: a.e. point $x \in \Omega_{t}$ lies in the relative interior of the distance ray of the set $\Omega_{t}$ that intersects $\partial \Omega_{t}$ at a point of $(2,1)$ differentiability of the surface $\Gamma$. Fix such $t$. Let $r \in\left(0, r_{0}\right]$. Let $y \in \partial \Omega_{t}$ be a point of $(2,1)$ differentiability of $\Gamma$. Let $\kappa^{t}$ be the curvature of $\partial \Omega_{t}$ at $y$, let denote $\kappa_{r}^{t}$ be the curvature of $\partial\left(\Omega_{t}\right)_{r}$ at the unique point $y \in \partial\left(\Omega_{t}\right)_{r}$ nearest to $x$, then

$$
\frac{1}{\kappa_{r}^{t}}=\frac{1}{\kappa^{t}}+r
$$


We calculate using properties of the function $a$ on the rays proved in the Step 1, equation (70), and Lemma 14 with $n=2$ :

$$
\begin{aligned}
\int_{\mathbf{R}^{2}} w \varphi d z= & \int_{\Omega_{t}} \varphi d z \\
= & \sum_{k=1}^{N} \int_{U_{k}}\left(\varphi \circ G_{k}\right)\left(\Psi_{k} \circ G_{k}\right) J_{2} G_{k} d x \\
= & \sum_{k=1}^{N} \int_{\tilde{U}_{k}} \Psi_{k}\left(G_{k}\left(x^{\prime}, r\right)\right) \int_{r}^{\tilde{\gamma}_{r, k}\left(x^{\prime}\right)}\left[\frac{d a(s-r)}{d s}\right. \\
& \left.+a(s-r) \frac{\kappa_{r}^{t}}{1-\kappa_{r}^{t} s}\right]\left(\varphi\left(G_{k}\left(x^{\prime}, s\right)\right) J_{2} G_{k}\left(x^{\prime}, s\right) d s d x^{\prime} .\right.
\end{aligned}
$$

In the last expression we integrate by parts and use (56), (71) and (58). Then we get

$$
\begin{aligned}
\int_{\mathbf{R}^{2}} w \varphi d z= & \sum_{k=1}^{N} \int_{\tilde{U}_{k}} \Psi_{k}\left(G_{k}\left(x^{\prime}, r\right)\right)\left[a(0) \varphi\left(G_{k}\left(x^{\prime}, r\right)\right) J_{2} G_{k}\left(x^{\prime}, r\right)\right. \\
& \left.+\int_{r}^{\tilde{\gamma}_{r, k}\left(x^{\prime}\right)} a(s-r) \frac{\partial \varphi\left(G_{k}\left(x^{\prime}, s\right)\right)}{\partial s} J_{2} G_{k}\left(x^{\prime}, s\right) d s\right] d x^{\prime} . \\
= & \int_{\hat{\partial}\left(\Omega_{t}\right)_{r}} \varphi\left(P_{r}^{t}(y)\right) \partial_{t} d_{\Omega_{t}}^{s}\left(P_{r}^{t}(y)\right)\left(1-r \kappa_{r}(y)\right) d \mathcal{H}^{1}(y)+\int_{\Omega_{t}} a D \varphi D u d z,
\end{aligned}
$$

where

$$
\hat{\partial}\left(\Omega_{t}\right)_{r}=\left\{y \in \partial\left(\Omega_{t}\right)_{r} \mid \gamma_{\left(\Omega_{t}\right)_{r}}(y)>r\right\}
$$

and

$$
P_{r}^{t}:\left(\Omega_{t}\right)_{r} \rightarrow \Omega_{t}
$$

is the nearest point projection mapping. By Proposition $9 \mathrm{~b}$ ) the mapping $P_{r}^{t}$ is well-defined and onto.

By Proposition 13, if $\Gamma_{r}$ is $(2,1)$ differentiable at $y \in \hat{\partial}\left(\Omega_{t}\right)_{r}$, then $\Gamma$ is $(2,1)$ differentiable at $y^{\prime}=P_{r}^{t}(y)$ and

$$
\partial_{t} d_{\Omega_{t}}^{s}\left(P_{r}^{t}(y)\right)=\partial_{t} d_{\Omega_{t}}^{s}(y)
$$

By the equality

$$
d_{\left(\Omega_{t}\right)_{r}}^{s}(x)=d_{\Omega_{t}}^{s}(x)+r \quad \text { for }(x, t) \in E_{r_{0}}, \quad r \in\left(0, r_{0}\right]
$$

we see that

$$
\partial_{t} d_{\Omega_{t}}^{s}\left(P_{r}^{t}(y)\right)=\partial_{t} d_{\left(\Omega_{t}\right)_{r}}^{s}(y)
$$

for $y \in \hat{\partial}\left(\Omega_{t}\right)_{r}$.

Thus we have showed that for a.e. $t \in[0, T]$, every $r \in\left(0, r_{0}\right]$ the equality holds

$$
\int_{\mathbf{R}^{2}} w \varphi d z=\int_{\hat{\partial}\left(\Omega_{t}\right)_{r}}\left(\varphi \circ P_{r}^{t}\right) \partial_{t} d_{\left(\Omega_{t}\right)_{r}}^{s}\left(1-r \kappa_{r}^{t}\right) d \mathcal{H}^{1}+\int_{\Omega_{t}} a D \varphi D u d z .
$$


In the next lemma we show that in the first integral at the right-hand side of (81) we can integrate over the whole boundary $\partial\left(\Omega_{t}\right)_{r}$.

Lemma 31. Let $r \in\left(0, r_{0}\right]$ be such that properties (70), (77), (78) are satisfied. Then for a.e. $t \in(0, T)$

$$
\int_{\partial\left(\Omega_{t}\right)_{r} \cap\left\{\gamma_{\left.\left(\Omega_{t}\right)_{r} \leq r\right\}}\right.} \varphi \partial_{t} d_{\left(\Omega_{t}\right)_{r}}^{s}\left(1-r \kappa_{r}^{t}\right) d \mathcal{H}^{1}=0
$$

Proof. We will show that for a.e. $t$

$$
\partial_{t} d_{\left(\Omega_{t}\right)_{r}}^{s}(y)\left(1-r \kappa_{r}^{t}(y)\right)=0 \quad \mathcal{H}^{1} \text { a.e. on } \partial\left(\Omega_{t}\right)_{r} \cap\left\{\gamma_{\left(\Omega_{t}\right)_{r}} \leq r\right\} .
$$

By (77) for a.e. $t$ the surface $\Gamma_{r}$ is $(2,1)$ differentiable $\mathcal{H}^{1}$ a.e. on $\partial\left(\Omega_{t}\right)_{r}$. Fix such $t$. Then in (82) we can consider only $y$ at which $\Gamma_{r}$ is $(2,1)$ differentiable. Fix such $y$. By Proposition 9,

$$
\gamma_{\left(\Omega_{t}\right)_{r}} \geq r \quad \text { on } \partial\left(\Omega_{t}\right)_{r}
$$

Thus

$$
\gamma_{\left(\Omega_{t}\right)_{r}}(y)=r
$$

It follows that

$$
\kappa_{r}^{t}(y) \leq \frac{1}{r}
$$

Let $y^{\prime}=P_{r}^{t}(y)$, i.e., $y^{\prime}$ is the point of $\partial \Omega_{t}$ nearest to $y$. Consider 3 cases.

Case 1. $y$ is the unique point of $\partial\left(\Omega_{t}\right)_{r}$ for which $y^{\prime}$ the nearest point on $\partial \Omega_{t}$.

Then the calculations of EGan, Proposition 7.1, Steps 2,3 imply that

$$
\kappa_{r}^{t}(y)=\frac{1}{r}
$$

Thus we have (82) in this case.

Case 2. There exists $z \in \partial\left(\Omega_{t}\right)_{r}, z \neq y$, such that $y^{\prime}=P_{r}^{t}(z)$, and

$$
B_{r}(y) \cap B_{r}(z) \neq \emptyset \text {. }
$$

Note that

$$
\left|y-y^{\prime}\right|=\left|z-y^{\prime}\right|=r .
$$

Then, denoting $w$ the point $\frac{y+z}{2}$, we get

$$
w \in B_{r}(y) \cup B_{r}(z) .
$$

We have

$$
B_{r}(y), B_{r}(z) \subset \mathbf{R}^{2} \backslash \bar{\Omega}_{t} .
$$

Thus $w \in \mathbf{R}^{2} \backslash \bar{\Omega}_{t}$, and $y^{\prime}$ is the point of $\partial \Omega_{t}$ nearest to $w$. Denote

$$
v=y^{\prime}+\frac{r}{\left|w-y^{\prime}\right|}\left(w-y^{\prime}\right)
$$

Then, by Condition 3 , we get the following:

$$
v \notin \Omega_{t}, \quad y^{\prime} \text { is the unique point of } \partial \Omega_{t} \text { nearest to } v \text {. }
$$

But

$$
\left|v-y^{\prime}\right|=r
$$


Thus we proved that

$$
v \in \partial\left(\Omega_{t}\right)_{r}
$$

The points $y$ and $z$ divide the circle $\partial B_{r}\left(y^{\prime}\right)$ on two arcs, and the point $v$ is the middle point of one of these arcs. Denote this $\operatorname{arc} C_{1}$. Repeating the above argument inductively and using continuity of distance function, we prove that

$$
C_{1} \subset \partial\left(\Omega_{t}\right)_{r} .
$$

But then

$$
\kappa_{r}^{t}(y)=\frac{1}{r}
$$

Thus we have (82) in the Case 2 .

Case 3. There exists $z \in \partial\left(\Omega_{t}\right)_{r}, z \neq y$, such that $y^{\prime}=P_{r}^{t}(z)$, and

$$
B_{r}(y) \cap B_{r}(z)=\emptyset .
$$

Then

$$
\partial B_{r}(y) \cap \partial B_{r}(z)=y^{\prime} .
$$

Since $\left\{\Omega_{t}\right\}$ is an expanding family of sets, we have

$$
B_{r}(y), B_{r}(z) \subset \mathbf{R}^{2} \backslash \bar{\Omega}_{\tau} \text { for } \tau<t .
$$

If

$$
y^{\prime} \in \partial \Omega_{\tau^{*}} \text { for some } \tau^{*}<t,
$$

then the same is true for all $\tau \in\left[\tau^{*}, t\right]$, and (83) implies that

$$
y \in \partial\left(\Omega_{\tau}\right)_{r} \quad \text { for all } \tau \in\left[\tau^{*}, t\right] .
$$

Then

$$
\partial_{t} d_{\left(\Omega_{t}\right)_{r}}^{s}(y)=0
$$

Thus we have (82) if (84) is satisfied.

The remaining case is

$$
y^{\prime} \notin \partial \Omega_{\tau} \quad \text { for all } \tau<t .
$$

Introduce a coordinate system $\left(x_{1}, x_{2}\right)$ on $R^{2}$ in which

$$
y^{\prime}=(0,0), \quad y=(0, r) \text {. }
$$

Then

$$
z=(0,-r) .
$$

Since $\left\{\Omega_{t}\right\}$ is a continuous expanding family of sets, the function

$$
\phi(\tau)=\operatorname{dist}\left(y^{\prime}, \Omega_{\tau}\right)
$$

is continuous and nonincreasing, and

$$
\phi(t)=0 .
$$

Let $w_{\tau} \in \mathcal{N}_{\partial \Omega_{\tau}}\left(y^{\prime}\right)$. Then by $(83)$

$$
\left.w_{\tau} \in \partial B_{\phi(\tau)}(0,0) \backslash\left[B_{r}(0, r) \cup B_{r}(0,-r)\right)\right] .
$$


In particular,

$$
w_{\tau} \rightarrow y^{\prime} \quad \text { as } \quad \tau \rightarrow t
$$

By Condition 3 we get

$$
B_{r}\left(v_{\tau}\right) \subset \mathbf{R}^{2} \backslash \bar{\Omega}_{\tau} \quad \text { where } \quad v_{\tau}=w_{\tau}+\frac{r}{\left|y^{\prime}-w_{\tau}\right|}\left(y^{\prime}-w_{\tau}\right) .
$$

By (86), (87) we see that there exists a sequence $\tau_{j} \rightarrow t$ such that

$$
v_{\tau_{j}} \rightarrow p \text {, where } p \text { is either }(-r, 0) \text { or }(r, 0) \text {. }
$$

Then by (88) and continuity of the family $\left\{\Omega_{t}\right\}$ we conclude

$$
\text { either } \quad B_{r}(-r, 0) \subset \mathbf{R}^{2} \backslash \bar{\Omega}_{t} \quad \text { or } \quad B_{r}(r, 0) \subset \mathbf{R}^{2} \backslash \bar{\Omega}_{t} .
$$

Let in fact

$$
B_{r}(r, 0) \subset \mathbf{R}^{2} \backslash \bar{\Omega}_{t} .
$$

Then $y^{\prime}=(0,0)$ is the point of $\partial \Omega_{t}$ nearest to the point $(r, 0)$. Thus

$$
(r, 0) \in \partial\left(\Omega_{t}\right)_{r} .
$$

But $y=(0, r)$, and thus

$$
B_{r}(y) \cap B_{r}(r, 0) \neq \emptyset .
$$

Thus the points $y$ and $(r, 0)$ satisfy the conditions of Case 2 . Thus we get

$$
\kappa_{r}^{t}(y)=\frac{1}{r} .
$$

Case 3 is proved. Then Lemma 31 is proved.

The properties (76), (77), (78) are satisfied for a.e. $r \in\left(0, r_{0}\right]$. Then there exists a decreasing sequence $r_{i} \rightarrow 0$, where $i=1,2, \ldots$ and $r_{i} \in\left(0, r_{0}\right]$, such that (76), (77), (78) are satisfied for each $r_{i}$. Note that by (78), the function

$$
(y, t) \rightarrow \kappa_{r_{i}}^{t}(y)
$$

defined on $\Gamma_{r_{i}}$ is $\mathcal{H}^{2}$ measurable.

Now we can integrate (81) by $t$ and use Lemma 31 to get

$$
\begin{aligned}
\int_{0}^{T} \int_{\mathbf{R}^{2}} w \varphi d z d t= & \int_{0}^{T} \int_{\partial\left(\Omega_{t}\right)_{r_{i}}}\left(\varphi \circ P_{r_{i}}^{t}\right) \partial_{t} d_{\left(\Omega_{t}\right)_{r_{i}}}^{s}\left(1-r_{i} \kappa_{r_{i}}^{t}\right) d \mathcal{H}^{1} d t \\
& +\int_{0}^{T} \int_{\Omega_{t}} a D \varphi D u d z d t .
\end{aligned}
$$

It follows that the first integral at the right-hand side does not depend on $r_{i}$. Thus it is enough to compute the limit as $i \rightarrow \infty$. We will prove the following:

$$
\lim _{i \rightarrow \infty} \int_{0}^{T} \int_{\partial\left(\Omega_{t}\right)_{r_{i}}}\left(\varphi \circ P_{r_{i}}^{t}\right) \partial_{t} d_{\left(\Omega_{t}\right)_{r_{i}}}^{s}\left(1-r_{i} \kappa_{r_{i}}^{t}\right) d \mathcal{H}^{1} d t=-\int_{0}^{T} \int_{\mathbf{R}^{2}} \chi_{\Omega_{t}} \partial_{t} \varphi d x d t
$$

We first prove that such equality is true if the boundary satisfies additional regularity assumptions. 
Lemma 32. Let $\left\{\Omega_{t}\right\}$ satisfy (76) - (78) with $r=0$. Let $V(x, t)$ be the outer normal velocity of $\Gamma_{t}$, defined by (6) at every point $(x, t)$ of differentiability of $\Gamma$ and defined as 0 at all points where $\Gamma$ is not differentiable. Then for every $\varphi \in C_{c}^{1}\left(\mathbf{R}^{2} \times(0, T)\right)$

$$
\int_{0}^{T} \int_{\partial \Omega_{t}} \varphi V d \mathcal{H}^{1} d t=-\int_{0}^{T} \int_{\mathbf{R}^{2}} \chi_{\Omega_{t}} \partial_{t} \varphi d x d t
$$

Proof. Let the set $E$ be defined by (5). Then by (76) $E$ has locally finite perimeter. By (77)

$$
\mathcal{H}^{2}\left(\partial E \backslash \partial_{*} E\right)=0
$$

where $\partial_{*} E$ is the reduced boundary of $E$. Let $\Phi: \mathbf{R}^{2} \times \mathbf{R}^{1} \rightarrow \mathbf{R}^{2} \times \mathbf{R}^{1}$ be defined by

$$
\Phi(x, t)=(0, \ldots, 0, \varphi(x, t))
$$

Let $\nu_{E}(x, t)$ be the measure-theoretical outer normal to $\Gamma$ at $(x, t) \in \Gamma$. Then by Green-Gauss theorem for sets with finite perimeter (EGar92, section 5.8)

$$
\int_{0}^{T} \int_{\mathbf{R}^{2}} \chi_{\Omega_{t}} \partial_{t} \varphi d x d t=\int_{E} \operatorname{div} \Phi d x d t=\int_{\Gamma} \Phi \nu_{E} d \mathcal{H}^{2},
$$

where

$$
\operatorname{div} \Phi=\sum_{i=1}^{3} \frac{\partial \Phi_{i}}{\partial x_{i}}, \quad \text { where } x_{3}=t .
$$

At every point $(x, t)$ of differentiability of $\Gamma$ we have

$$
\nu_{E}(x, t)=-D_{(x, t)} d_{E}^{s}(x, t),
$$

where $d_{E}^{s}$ is the signed distance to the boundary of the set $E$ in the $(x, t)$-space. Thus we get

$$
\int_{E} \partial_{t} \varphi d x d t=-\int_{\Gamma} \varphi \partial_{t} d_{E}^{s} d \mathcal{H}^{2}
$$

Let $f: \Gamma \rightarrow \mathbf{R}^{1}$ be the mapping defined by

$$
f(x, t)=t
$$

Then $f$ is Lipschitz, and $f$ and $\Gamma$ are differentiable at $\mathcal{H}^{2}$ a.e. point $(x, t) \in \Gamma$. At such point $(x, t)$ the gradient of $f$ is a linear mapping $D f(x, t): T_{x, t} \Gamma \rightarrow \mathbf{R}^{1}$, where $T_{x, t} \Gamma$ is the tangent to $\Gamma$ at $(x, t)$ space. Let $e_{1}, e_{2}$ be such orthonormal basis in $\mathbf{R}^{2}$ that $e_{2}$ is the inner normal to $\partial \Omega_{t}$ at $x$. Let $\tau$ be the unit vector in the $t$-direction. Then the vectors

$$
e_{1}, \tilde{e}_{2}=-\partial_{t} d_{E}^{s}(x, t) e_{2}+\left|D_{x} d_{E}^{s}(x, t)\right| \tau
$$

form an orthonormal basis in $T_{x, t} \Gamma$. We calculate:

$$
\begin{aligned}
& D f(x, t) e_{1}=0, \\
& D f(x, t) \tilde{e}_{2}=\left|D_{x} d_{E}^{s}(x, t)\right| .
\end{aligned}
$$

Thus

$$
|D f(x, t)|=\left|D_{x} d_{E}^{s}(x, t)\right| .
$$


Also, the following relation holds:

$$
D_{x, t} d_{\Omega_{t}}^{s}(x, t)=\frac{1}{\left|D_{x} d_{E}^{s}(x, t)\right|} D_{x, t} d_{E}^{s}(x, t) .
$$

Now, applying formula 3.2.22 of [Fed69] (which is applicable by (76)), we get:

$$
\begin{aligned}
\int_{\Gamma} \varphi \partial_{t} d_{E}^{s} d \mathcal{H}^{2} & =\int_{\Gamma} \varphi \partial_{t} d_{\Omega_{t}}^{s}|D f(x, t)| d \mathcal{H}^{2} \\
& =\int_{0}^{T} \int_{f^{-1}(t)} \varphi \partial_{t} d_{\Omega_{t}}^{s} d \mathcal{H}^{1} d t \\
& =\int_{0}^{T} \int_{\partial \Omega_{t}} \varphi V d \mathcal{H}^{1} d t .
\end{aligned}
$$

The lemma is proved.

Now we can prove (90). Each $\left\{\left(\Omega_{t}\right)_{r_{i}}\right\}$ satisfies (76) - (78). Thus we have

$$
\int_{0}^{T} \int_{\partial\left(\Omega_{t}\right)_{r_{i}}} \varphi V^{\left(r_{i}\right)} d \mathcal{H}^{1} d t=-\int_{0}^{T} \int_{\mathbf{R}^{2}} \chi_{\left(\Omega_{t}\right)_{r_{i}}} \partial_{t} \varphi d x d t
$$

where $V^{r_{i}}$ is velocity of $\left(\Omega_{t}\right)_{r_{i}}$. It follows from Proposition 21 that $\mathcal{L}^{3}(\Gamma)=0$, and thus

$$
\chi_{\left(\Omega_{t}\right)_{r_{i}}}(x) \rightarrow \chi_{\Omega_{t}}(x) \quad \text { for a.e. }(x, t) \in \mathbf{R}^{n} \times(0, T) .
$$

Then by Dominated Convergence Theorem the right-hand side of (92) converges to the right-hand side of (90) as $i \rightarrow \infty$. Thus it remains to prove that the left-hand side of (92) and the left-hand side of (90) converge to the same limit.

Let $P_{r, r_{i}}^{t}: \partial\left(\Omega_{t}\right)_{r} \rightarrow \partial\left(\Omega_{t}\right)_{r_{i}}$ be the nearest point projection (well-defined by Proposition 9). $P_{r, r_{i}}^{t}$ is a Lipschitz map by Propositions 9, 11. Then using Lemma 14 and identity (23), the difference between the left-hand sides of (92) and (90) can be transformed to

$$
\begin{aligned}
& \int_{0}^{T} \int_{\partial\left(\Omega_{t}\right)_{r}}\left(\varphi \circ P_{r, r_{i}}^{t}-\varphi \circ P_{r}^{t}\right) V^{r_{i}} \circ P_{r, r_{i}}^{t}\left(1-r \kappa_{r}^{t}\right) d \mathcal{H}^{1} d t+ \\
& r_{i} \int_{0}^{T} \int_{\partial\left(\Omega_{t}\right)_{r}} \varphi \circ P_{r, r_{i}}^{t} V^{r_{i}} \circ P_{r, r_{i}}^{t} \kappa_{r}^{t} d \mathcal{H}^{1} d t=I_{1, i}+r_{i} I_{2, i} .
\end{aligned}
$$

We have $\left|V^{r_{i}}\right|<C$, where $C$ does not depend on $i$, and $\left|\kappa_{r}^{t}\right| \leq \frac{1}{r}$. Using the fact that $P_{r, r_{i}}^{t} \rightarrow P_{r}^{t}$ as $r_{i} \rightarrow 0$ and Dominated Convergence Theorem we see that $I_{1, i} \rightarrow 0$. We also have $\left|I_{2, i}\right|<C$. Thus the expression (93) converges to zero. Thus (90) is proved.

The equalities (89) and (90) imply (75).

Step 3.

The family $\left\{\Omega_{t}\right\}$ is expanding. Thus the left-hand side of (90) defines a nonnegative linear functional of $\varphi \in C^{\infty}\left(\mathbf{R}^{n} \times[0, T]\right)$. By EGar92, Chapter 1.8, Corollary 1, it 
follows from (90) that $\partial_{t} \chi_{\Omega_{t}}$ is a nonnegative Radon measure. Thus the mass balance equation (75) can be rewritten as

$$
\int_{0}^{T} \int_{\mathbf{R}^{2}} w \varphi d x t d t+\int_{\mathbf{R}^{2} \times(0, T)} \varphi d w_{t}-\int_{0}^{T} \int_{\mathbf{R}^{2}} a D u D \varphi d x t d t=0 .
$$

From this equation it follows that for a.e. $t \in[0, T]$, every $\varphi \in C_{c}^{1}\left(\mathbf{R}^{2}\right)$

$$
\int_{\mathbf{R}^{2}} w(\cdot, t) \varphi d x+\int_{\mathbf{R}^{2}} \varphi d w_{t}(\cdot, t)-\int_{\mathbf{R}^{2}} a(\cdot, t) D u(\cdot, t) D \varphi d x=0 .
$$

Now, repeating argument of Section 6, we conclude the proof of Theorem 30 .

\section{Appendix A. Appendix}

Let $\Omega$ be an open set. We write $d(x)$ for $d_{\Omega}(x)$ below. The purpose of this section is to prove that the gradient of $d_{\Omega}$ is locally Lipschitz at $x \in \Omega \backslash \mathcal{R}$ and give an estimate of the Lipschitz constant in the terms of the distance between $x$ and endpoints of the distance ray $R_{x}$.

Proposition 33 should be compared with Proposition 4.1 of EGan and with inequality $4.8(8)$ of [Fed59].

In the inequality (95) below the only assumption regarding the point $x_{1}$ is that it is close enough to $x$. In particular it is possible that $x_{1} \in \mathcal{R}$. In the inequality $4.8(8)$ of [Fed59] the conditions on $x$ and $x_{1}$ are symmetric and exclude the possibility that $x_{1} \in \mathcal{R}$.

Proposition 33 improves the estimates of Proposition 4.1 of [EGan in the following. Two quantities are estimated explicitly in Proposition 33: the local Lipschitz constant of $\operatorname{Dd}(\cdot)$ at $x$ and the size of the neighborhood of $x$ in which the estimates (95) and (96) hold.

Proposition 33. There exist constants $C$ and $M$ depending only on $n$ such that the following is true. Let $\Omega \in R^{n}$ be an open set. Let $\varepsilon>0, x \in \Omega \backslash \mathcal{R}$, and let

$$
d_{\Omega}(x) \geq M \varepsilon, \quad \gamma_{\Omega}(x)-d_{\Omega}(x) \geq M \varepsilon .
$$

Then by Remark 0 there exists a unique ray $R_{x}$. Denote $y$ and $v$ the lower and upper ends of $R_{x}$, i.e., $y=R_{x} \cap \partial \Omega$ and $v=R_{x} \cap \mathcal{R}$. Then for every $x_{1} \in \Omega$ satisfying $\left|x-x_{1}\right|<\varepsilon$, the inequality holds

$$
\left|y-y_{1}\right| \leq C\left(1+\frac{|x-y|}{|x-v|}\right)\left|x-x_{1}\right|,
$$

where $y_{1} \in \mathcal{N}_{\partial \Omega}\left(x_{1}\right)$. If in addition the function $d(\cdot)$ is differentiable at $x_{1}$ then

$$
\left|\operatorname{Dd}(x)-\operatorname{Dd}\left(x_{1}\right)\right| \leq \frac{C}{\varepsilon}\left|x-x_{1}\right| .
$$

Proof. We first prove the inequalities (95), (96) assuming that

$$
|x-v| \geq|x-y| \text {. }
$$

Denote $d:=d_{\Omega}(x)$. By (97), we can find a point $O$ on the interval of $R_{x}$ between $v$ and $x$ such that $|x-O|=d$. Let $y \in \mathcal{N}_{\partial \Omega}(x)$, then we also have $y \in \mathcal{N}_{\partial \Omega}(O)$. 
In the calculations below $C$ will denote different constants depending only on $n$. We assume that

$$
M>10 .
$$

Choose $x_{1} \in B_{\varepsilon}(x)$. Let $\hat{x}_{1}$ be projection of $x_{1}$ onto $R_{x}$, then

$$
\left|x-\hat{x}_{1}\right| \leq \varepsilon \leq \frac{1}{10} d
$$

by (94), (98). Thus $\hat{x}_{1}$ lies between $y$ and $O$ on $R_{x}$. Let $d_{1}=\left|\hat{x}_{1}-O\right|$, then

$$
\frac{11}{10} \geq \frac{d}{d_{1}} \geq \frac{9}{10}, \quad\left|d-d_{1}\right|<\varepsilon
$$

Introduce the following coordinate system in $R^{n}$ : let the point $O$ be the origin, let $e_{n}=\frac{x-O}{|x-O|}$ (thus $e_{n}$ is the unit vector along the ray $R_{x}$ ), let $e_{1}, \ldots, e_{n-1}, e_{n}$ be an orthonormal basis in $\mathbf{R}^{n}$. Then in these coordinates

$$
x=(0, d), \quad \hat{x}_{1}=\left(0, d_{1}\right), \quad y=(0,2 d),
$$

where $0 \in \mathbf{R}^{n-1}$. We also have $x_{1}=\left(x_{1}^{\prime}, d_{1}\right)$, where $x_{1}^{\prime} \in \mathbf{R}^{n-1}$. Let $y_{1} \in \partial \Omega$ be such that $\left|x_{1}-y_{1}\right|=d\left(x_{1}\right)$. Let the coordinates of $y_{1}$ be $\left(y_{1}^{\prime}, y_{1, n}\right)$ where $y_{1}^{\prime} \in \mathbf{R}^{n-1}$, $y_{1, n} \in \mathbf{R}^{1}$. Then

$$
\left|x_{1}-y_{1}\right| \leq\left|x_{1}-y\right|
$$

or

$$
\left|x_{1}^{\prime}-y_{1}^{\prime}\right|^{2}+\left|d_{1}-y_{1, n}\right|^{2} \leq\left|x_{1}^{\prime}\right|^{2}+\left|d_{1}-2 d\right|^{2}
$$

Claim 34. There exists $M_{1}$, depending on $n$, such that for any $\delta \in(0,2)$ the following is true:

$$
\text { if } d>\frac{M_{1}}{\delta^{2}} \varepsilon \text { then }\left|y_{1}^{\prime}\right|<\delta d
$$

Proof. Note that

$$
\left|x_{1}^{\prime}\right|<\varepsilon, \quad\left|y_{1}^{\prime}\right|<2 d .
$$

Indeed, the first inequality is true since $x_{1} \in B_{\varepsilon}(x)$. To prove the second inequality of (103) we use (101) and (99) to get

$$
\left|y_{1}^{\prime}-x_{1}^{\prime}\right|^{2} \leq \varepsilon^{2}+|d+\varepsilon|^{2}<(d+\sqrt{2} \varepsilon)^{2},
$$

so

$$
\left|y_{1}^{\prime}\right| \leq\left|x_{1}^{\prime}\right|+d+\sqrt{2} \varepsilon<d+3 \varepsilon<2 d
$$

since $d \geq 10 \varepsilon$. Thus (103) is proved.

Suppose that the assertion of Claim 34 is false, that is

$$
\left|y_{1}^{\prime}\right| \geq \delta d \text {. }
$$

By (102)

$$
\delta d>\frac{M_{1}}{\delta} \varepsilon>2 \varepsilon
$$


if $M_{1}>4$. Then we get from (101), (103), (99), (105)

$$
\begin{aligned}
\left(y_{1, n}-d_{1}\right)^{2} & \leq \varepsilon^{2}+\left(2 d-d_{1}\right)^{2}-\left(\left|y_{1}^{\prime}\right|-\left|x_{1}^{\prime}\right|\right)^{2} \\
& \leq \varepsilon^{2}+\left(\frac{11}{10} d\right)^{2}-(\delta d-\varepsilon)^{2} \\
& <4 d^{2}\left(1-\frac{\delta^{2}}{4}\right)-2 d^{2}+2 \delta d \varepsilon \\
& \leq 4 d^{2}\left(1-\frac{\delta^{2}}{4}\right)
\end{aligned}
$$

The last inequality follows from $-2 d^{2}+2 \delta d \varepsilon<0$ which holds by (102) if $\delta<2$ and $M_{1}>8$.

Consider two cases:

Case 1: $y_{1, n} \geq d_{1}$. Denote by $\alpha$ the angle between vectors $y_{1}-x_{1}$ and $\left(x_{1}^{\prime}, y_{1, n}\right)-x_{1}$. In the triangle $x_{1}, y_{1},\left(x_{1}^{\prime}, y_{1, n}\right)$ the side $\left(x_{1}^{\prime}, y_{1, n}\right)-y_{1}$ is orthogonal to $x_{1}-\left(x_{1}^{\prime}, y_{1, n}\right)$, since $\left(x_{1}^{\prime}, y_{1, n}\right)-y_{1}=\left(x_{1}^{\prime}-y_{1}^{\prime}, 0\right)$, and $x_{1}-\left(x_{1}^{\prime}, y_{1, n}\right)=\left(0, d_{1}-y_{1, n}\right)$. Thus we get

$$
\tan \alpha=\frac{\left|y_{1}^{\prime}-x_{1}^{\prime}\right|}{y_{1, n}-d_{1}}
$$

But

$$
\left|y_{1}^{\prime}-x_{1}^{\prime}\right| \geq|| y_{1}^{\prime}|-| x_{1}^{\prime}||=|\delta d-\varepsilon|=\delta d-\varepsilon
$$

by $(106)$. By (107) we get

$$
\tan \alpha \geq \frac{\delta d-\varepsilon}{2 d \sqrt{1-\frac{\delta^{2}}{4}}}=\frac{\delta}{2 \sqrt{1-\frac{\delta^{2}}{4}}}\left(1-\frac{1}{\delta} \cdot \frac{\varepsilon}{d}\right) \geq \frac{\delta}{4 \sqrt{1-\frac{\delta^{2}}{4}}} .
$$

where the last inequality follows from (106). Thus, using the condition $\delta \in(0,2)$ we get

$$
|\cos \alpha|=\frac{1}{\sqrt{1+\tan ^{2} \alpha}} \leq \sqrt{\frac{4\left(4-\delta^{2}\right)}{16-3 \delta^{2}}} \leq 1-\frac{\delta^{2}}{2\left(16-3 \delta^{2}\right)} .
$$

Consider the triangle $x_{1}, y_{1},\left(x_{1}^{\prime}, 0\right)$. The angle between the vectors $\left(x_{1}^{\prime}, 0\right)-x_{1}$ and $y_{1}-x_{1}$ is $\pi-\alpha$, and so we get

$$
\left|y_{1}-\left(x_{1}^{\prime}, 0\right)\right|^{2}=\left|x_{1}-\left(x_{1}^{\prime}, 0\right)\right|^{2}+\left|y_{1}-x_{1}\right|^{2}-2\left|x_{1}-\left(x_{1}^{\prime}, 0\right)\right|\left|y_{1}-x_{1}\right| \cos (\pi-\alpha) .
$$

Since $x_{1}=\left(x_{1}^{\prime}, d_{1}\right)$, we have

$$
\left|x_{1}-\left(x_{1}^{\prime}, 0\right)\right|=d_{1} \text {. }
$$

From (101) we get

$$
\left|y_{1}-x_{1}\right| \leq \sqrt{\varepsilon^{2}+\left(2 d-d_{1}\right)^{2}} .
$$


Then by (109), (108) we get

$$
\begin{aligned}
\left|y_{1}-\left(x_{1}^{\prime}, 0\right)\right|^{2} & \leq d_{1}^{2}+\left[\varepsilon^{2}+\left(2 d-d_{1}\right)^{2}\right]+2 d_{1} \sqrt{\varepsilon^{2}+\left(2 d-d_{1}\right)^{2}}\left(1-\frac{\delta^{2}}{32}\right) \\
& \leq d_{1}^{2}+\left(2 d-d_{1}\right)^{2}+2 d_{1}\left(2 d-d_{1}\right)\left(1+\frac{\varepsilon^{2}}{2\left(2 d-d_{1}\right)^{2}}\right)\left(1-\frac{\delta^{2}}{32}\right)+\varepsilon^{2} \\
& \leq 4 d^{2}+2 d_{1} \frac{\varepsilon^{2}\left(1-\frac{\delta^{2}}{32}\right)}{2\left(2 d-d_{1}\right)}+\varepsilon^{2}-2 d_{1}\left(2 d-d_{1}\right) \frac{\delta^{2}}{32} \\
& \leq 4 d^{2}+d_{1} \frac{\varepsilon^{2}}{2 d-d_{1}}+\varepsilon^{2}-\frac{1}{16} d_{1}\left(2 d-d_{1}\right) \delta^{2} .
\end{aligned}
$$

Thus we see that

$$
\left|y_{1}-\left(x_{1}^{\prime}, 0\right)\right|^{2}<(2 d-\varepsilon)^{2},
$$

provided

$$
d_{1} \frac{\varepsilon^{2}}{2 d-d_{1}}-\frac{1}{16} d_{1}\left(2 d-d_{1}\right) \delta^{2}<-4 d \varepsilon .
$$

Using (99) we see that (111) is satisfied if

$$
d^{2} \delta^{2}>C\left(\varepsilon^{2}+d \varepsilon\right)
$$

where $C$ is a large enough constant. In its turn, (112) is true if $d>\frac{M_{1}}{\delta^{2}} \varepsilon$, where $M_{1}$ is large enough. Thus, for such $d$, the inequality (110) holds, and so

$$
\left|y_{1}-\left(x_{1}^{\prime}, 0\right)\right|<2 d-\varepsilon \text {. }
$$

Then

$$
\left|y_{1}\right| \leq\left|x_{1}^{\prime}\right|+\left|y_{1}-\left(x_{1}^{\prime}, 0\right)\right|<2 d=|y| .
$$

This contradicts the fact that $y \in \mathcal{N}_{\partial \Omega}(O)$. Thus (105) is false. Thus Case 1 of Claim 34 is proved.

Case 2: $y_{1, n} \leq d_{1}$.

Using this condition, we get

$$
\begin{aligned}
\left|\left(x_{1}^{\prime}, 0\right)-y_{1}\right|^{2}= & \left|\left(x_{1}^{\prime}, 0\right)-x_{1}+x_{1}-y_{1}\right|^{2}=\left|-\left(0, d_{1}\right)+\left(x_{1}^{\prime}-y_{1}^{\prime}, d_{1}-y_{1, n}\right)\right|^{2} \\
= & d_{1}^{2}+\left|x_{1}-y_{1}\right|^{2}+2\left(y_{1, n}-d_{1}\right) d_{1} \\
\leq & d_{1}^{2}+\left|x_{1}-y_{1}\right|^{2} \leq d_{1}^{2}+\varepsilon^{2}+\left(2 d-d_{1}\right)^{2} \\
= & 4 d^{2}-2 d_{1}\left(2 d-d_{1}\right)+\varepsilon^{2}<(2 d-\varepsilon)^{2}, \\
& \text { if } d_{1}\left(2 d-d_{1}\right)>2 d \varepsilon .
\end{aligned}
$$

The last inequality is in fact true by (99) and $d>10 \varepsilon$. Then, as in the Case 1 , we arrive to a contradiction. Thus Case 2 cannot happen for $d$ and $\varepsilon$ defined in Claim 34. Thus, Claim 34 is proved.

Now the rest of the proof of part (a) of Proposition 11 follows the proof of Proposition 4.1 in EGan. 
The main step is to show that there exist constants $C$ and $M$ depending only on $n$ such that the inequality $d>M \varepsilon$ implies

$$
\left|y-y_{1}\right| \leq C\left|\hat{x}_{1}-x_{1}\right| \text {. }
$$

Let $\delta \in(0,1)$ be chosen later, and let $d>\frac{M_{1}}{\delta^{2}} \varepsilon$ where $M_{1}$ is the constant from Claim 34. Then by Claim 34 we get

$$
\left|y_{1}^{\prime}\right|<\delta d
$$

Since $y \in \mathcal{N}_{\partial \Omega}(O)$, we get $\left|y_{1}\right|>|y|=2 d$, and so

$$
\left|y_{1}^{\prime}\right|^{2}+\left|y_{1, n}\right|^{2} \geq 4 d^{2} \text {. }
$$

Thus we estimate using (114)

$$
\begin{aligned}
y_{1, n} & \geq \sqrt{4 d^{2}-\left|y_{1}^{\prime}\right|^{2}}=2 d \sqrt{1-\frac{\left|y_{1}^{\prime}\right|^{2}}{4 d^{2}}} \geq 2 d\left(1-\frac{\left|y_{1}^{\prime}\right|^{2}}{8 d^{2}}-C \frac{\left|y_{1}^{\prime}\right|^{4}}{d^{4}}\right) \\
& \geq 2 d-\frac{\left|y_{1}^{\prime}\right|^{2}}{4 d}-C \frac{\left|y_{1}^{\prime}\right|^{4}}{4 d^{3}} \geq 2 d-\frac{\left|y_{1}^{\prime}\right|^{2}}{4 d}\left(1+C \delta^{2}\right),
\end{aligned}
$$

where all constants $C$ do not depend on $y_{1}^{\prime}$ and $d$. So

$$
0<2 d-d_{1}=y_{1, n}-d_{1}+2 d-y_{1, n} \leq\left(y_{1, n}-d_{1}\right)+\frac{\left|y_{1}^{\prime}\right|^{2}}{4 d}\left(1+C \delta^{2}\right) .
$$

Now we can estimate

$$
\begin{aligned}
\left(2 d-d_{1}\right)^{2} & \leq\left(y_{1, n}-d_{1}\right)^{2}+\left(y_{1, n}-d_{1}\right) \frac{\left|y_{1}^{\prime}\right|^{2}}{2 d}\left(1+C \delta^{2}\right)+\frac{\left|y_{1}^{\prime}\right|^{4}}{16 d^{2}}\left(1+C \delta^{2}\right) \\
& \leq\left(y_{1, n}-d_{1}\right)^{2}+\left(y_{1, n}-d_{1}\right) \frac{\left|y_{1}^{\prime}\right|^{2}}{2 d}\left(1+C \delta^{2}\right)+C \delta^{2}\left|y_{1}^{\prime}\right|^{2}
\end{aligned}
$$

where we again used (114). Using (101), (99) and (103) we get the following estimate

$$
\begin{aligned}
\left|y_{1, n}-d_{1}\right| & \leq \sqrt{\varepsilon^{2}+\left(2 d-d_{1}\right)^{2}}=\left(2 d-d_{1}\right) \sqrt{1+\frac{\varepsilon^{2}}{\left(2 d-d_{1}\right)^{2}}} \\
& \leq\left(2 d-d_{1}\right)\left(1+\frac{\varepsilon^{2}}{2\left(2 d-d_{1}\right)^{2}}\right) \leq\left(2 d-d_{1}\right)\left(1+C \frac{\varepsilon^{2}}{d^{2}}\right) \\
& \leq\left(2 d-d_{1}\right)\left(1+C \delta^{4}\right) \quad \text { since } d \geq \frac{M_{1}}{\delta^{2}} \varepsilon .
\end{aligned}
$$

Thus, by (116):

$$
\begin{aligned}
\left(2 d-d_{1}\right)^{2} & \leq\left(y_{1, n}-d_{1}\right)^{2}+\left(2 d-d_{1}\right) \frac{\left|y_{1}^{\prime}\right|^{2}}{2 d}\left(1+C \delta^{4}\right)+C\left|y_{1}^{\prime}\right|^{2} \delta^{2} \\
& \leq\left(y_{1, n}-d_{1}\right)^{2}+\Theta\left|y_{1}^{\prime}\right|^{2}
\end{aligned}
$$

where

Using (99) we estimate

$$
\Theta=\left(1-\frac{d_{1}}{2 d}\right)\left(1+C \delta^{4}\right)+C \delta^{2}
$$

$$
\Theta \leq \frac{11}{20}\left(1+C \delta^{4}\right)+C \delta^{2}<1
$$


if $\delta>0$ is small enough. Fix such $\delta$. Let $M_{1}$ be the constant from Claim 34 defined by this $\delta$. Let $M=\frac{M_{1}}{\delta^{2}}$ be our choice of the constant $M$ in the inequalities (94). Then the inequalities (94) imply (117) with $\Theta<1$. Thus (117) and (101) give:

$$
\left|y_{1}^{\prime}-x_{1}^{\prime}\right|^{2} \leq\left|x_{1}^{\prime}\right|^{2}+\Theta\left|y_{1}^{\prime}\right|^{2} \text {. }
$$

Now we estimate:

$$
\left|y_{1}^{\prime}\right| \leq\left|y_{1}^{\prime}-x_{1}^{\prime}\right|+\left|x_{1}^{\prime}\right|
$$

and from this, using (118), we get

$$
\begin{aligned}
\left|y_{1}^{\prime}\right|^{2} & \leq\left|y_{1}^{\prime}-x_{1}^{\prime}\right|^{2}+2\left|y_{1}^{\prime}-x_{1}^{\prime}\right|\left|x_{1}^{\prime}\right|+\left|x_{1}^{\prime}\right|^{2} \\
& \leq(1+\mu)\left|y_{1}^{\prime}-x_{1}^{\prime}\right|^{2}+\left(1+\frac{C}{\mu}\right)\left|x_{1}^{\prime}\right|^{2} \\
& \leq(1+\mu)\left(\left|x_{1}^{\prime}\right|^{2}+\Theta\left|y_{1}^{\prime}\right|^{2}\right)+\left(1+\frac{C}{\mu}\right)\left|x_{1}^{\prime}\right|^{2} .
\end{aligned}
$$

Choose $\mu>0$ so small that $(1+\mu) \Theta<1$, then we get

$$
\left|y_{1}^{\prime}\right| \leq C\left|x_{1}^{\prime}\right|=C\left|x_{1}-\hat{x}_{1}\right| \text {. }
$$

It remains to estimate $\left|y_{1, n}-y_{n}\right|$ where $y_{n}=2 d$.

From (115) we get

$$
2 d-y_{1, n} \leq C \frac{\left|y_{1}^{\prime}\right|^{2}}{d} \leq C\left|y_{1}^{\prime}\right|,
$$

since $\left|y_{1}^{\prime}\right|<\delta d$.

Since $x_{1}=\left(x_{1}^{\prime}, d_{1}\right), d\left(x_{1}\right)=\left|x_{1}-y_{1}\right|$, and $\left.d\left(\hat{x}_{1}\right)\right)=\left|\hat{x}_{1}-y\right|=2 d-d_{1}$, we calculate (using the fact that $\operatorname{Lip}[d(\cdot)]=1$ )

$$
y_{1, n}-d_{1} \leq\left|y_{1}-x_{1}\right|=2 d-d_{1}+d\left(x_{1}\right)-d\left(\hat{x}_{1}\right) \leq 2 d-d_{1}+\left|x_{1}-\hat{x}_{1}\right|
$$

and so

$$
y_{1, n}-2 d \leq\left|x_{1}-\hat{x}_{1}\right| .
$$

The estimates (119), (120), and (121) imply (113).

Now since

$$
\operatorname{Dd}\left(x_{1}\right)=\frac{x_{1}-y_{1}}{\left|x_{1}-y_{1}\right|}, \quad \operatorname{Dd}\left(\hat{x}_{1}\right)=\frac{\hat{x}_{1}-y}{\left|\hat{x}_{1}-y\right|},
$$

we get the following estimate

$$
\left|D d\left(x_{1}\right)-D d\left(\hat{x}_{1}\right)\right| \leq \frac{\left|x_{1}-\hat{x}_{1}\right|+\left|y_{1}-y\right|}{\left|\hat{x}_{1}-y\right|},
$$

then using the equality $\left|\hat{x}_{1}-y\right|=2 d-d_{1}$ and the inequalities (99) and (117) we deduce that

$$
\left|D d\left(x_{1}\right)-D d\left(\hat{x}_{1}\right)\right| \leq \frac{C}{d}\left|x_{1}-\hat{x}_{1}\right| .
$$

Note that $D d\left(\hat{x}_{1}\right)=D d(x)$ since the points $x$ and $\hat{x}_{1}$ are on the same ray $R_{x}^{1}$. Now from $\left|\hat{x}_{1}-x_{1}\right| \leq\left|x-x_{1}\right|$, we get (96) in the case of (97). Note also that (113) and inequality $\left|\hat{x}_{1}-x_{1}\right| \leq\left|x-x_{1}\right|$ imply (95). 

Let

In the case $|x-v|<|x-y|$ we can make the following reduction to the case (97).

$$
Q=|x-y|-|x-v|,
$$

and let $\tilde{\Omega}$ be the set $\{z \in \Omega \quad \operatorname{dist}(z, \partial \Omega)>Q\}$. Then $B_{\varepsilon}(x) \subset \tilde{\Omega}$ since the function $\operatorname{dist}(\cdot, \partial \Omega)$ is Lipschitz with constant 1 . For $z \in \tilde{\Omega}$ we have

$$
\operatorname{dist}(z, \partial \Omega))=\operatorname{dist}(z, \partial \tilde{\Omega})+Q .
$$

To see this consider $z_{1} \in \mathcal{N}_{\partial \Omega}(z)$, and let $z_{2}$ be the point of intersection of the interval connecting $z$ and $z_{1}$ with $\partial \tilde{\Omega}$. Then $z_{1} \in \mathcal{N}_{\partial \Omega}\left(z_{2}\right)$ since $z_{2}$ lies in the relative interior of the distance ray $R_{z_{1}}$ that intersects $\partial \Omega$ in $z_{1}$. Thus $\left|z_{1}-z_{2}\right|=Q$. Now suppose that there exists a point $\hat{z}_{2} \in \partial \tilde{\Omega}$ such that $\left|z-\hat{z}_{2}\right|<\left|z-z_{2}\right|$. Let $\hat{z}_{1} \in \mathcal{N}_{\partial \Omega}\left(\hat{z}_{2}\right)$, then $\left|\hat{z}_{1}-\hat{z}_{2}\right|=Q$. Then

$$
\left|z-\hat{z}_{1}\right| \leq\left|z-\hat{z}_{2}\right|+\left|\hat{z}_{2}-\hat{z}_{1}\right|<\left|z-z_{2}\right|+Q=\left|z-z_{1}\right|
$$

a contradiction to the fact that $z_{1} \in \mathcal{N}_{\partial \Omega}(z)$. This proves (123). So the distance rays and the ridge set of the set $\tilde{\Omega}$ are intersections of those of the set $\Omega$ with the set $\tilde{\Omega}$.

Then in (96) we can consider $d(\cdot)$ as the distance to $\partial \tilde{\Omega}$, so we can consider $\tilde{\Omega}$ instead of $\Omega$, and then the inequality (97) is satisfied.

To show (95), denote by $w$ and $w_{1}$ the points of intersection of $\partial \tilde{\Omega}$ with the intervals $(x, y)$ and $\left(x_{1}, y_{1}\right)$ respectively. Then it follows from (123) $w \in \mathcal{N}_{\partial \tilde{\Omega}}(x)$ and $w_{1} \in$ $\mathcal{N}_{\partial \tilde{\Omega}}\left(x_{1}\right)$, and

$$
|w-y|=\left|w_{1}-y_{1}\right|=Q .
$$

Then it follows from (122) that

$$
|x-v|=|x-w| .
$$

Using (122) and letting $A=\frac{|x-y|}{|x-v|}$, we get

$$
\begin{aligned}
y & =x+A(w-x), \\
y_{1} & =x_{1}+\left[1+(A-1) \frac{|x-w|}{\left|x_{1}-w_{1}\right|}\right]\left(w_{1}-x_{1}\right) .
\end{aligned}
$$

The inequality (95) can be applied to the set $\tilde{\Omega}$, so

$$
\left|w-w_{1}\right| \leq C\left|x-x_{1}\right| .
$$

Now we estimate:

$$
\begin{aligned}
\left|y-y_{1}\right|= & \mid x-x_{1}+A\left[(w-x)-\frac{|x-w|}{\left|x_{1}-w_{1}\right|}\left(w_{1}-x_{1}\right)\right] \\
& +\left(\frac{|x-w|}{\left|x_{1}-w_{1}\right|}-1\right)\left(w_{1}-x_{1}\right) \mid \\
\leq & C(1+A)\left(\left|x-x_{1}\right|+\left|w-w_{1}\right|\right) \\
\leq & C(1+A)\left|x-x_{1}\right| .
\end{aligned}
$$

This implies (95). 


\section{ACKNOWLEDGEMENT}

It is the author's pleasure to thank L. C. Evans and R. F. Gariepy for their interest, insight and and encouragement.

\section{REFERENCES}

[Ar95] G. Aronsson. Asymptotic solutions of a compression molding problem, Preprint LiTH-MATH-R-95-01, 1995, Department of Mathematics, Linkoping University, Linkoping, Sweden.

[AE] G. Aronsson, L. C. Evans. An asymptotic model for compression molding, Forthcoming.

[AEW96] G. Aronsson, L. C. Evans, Y. Wu. Fast/Slow diffusion and growing sandpiles, J.Diff.Equat., 131 (1996), no. 2, 304-335.

[BDM89] T. Bhattacharya, E. DiBenedetto, J. Manfredi. Limits as $p \rightarrow \infty$ of $\Delta_{p} u=f$ and related extremal problems, Rend. Sem. Mat. Univ. Politee, Torino, 1989.

[EFG97] L. C. Evans, M. Feldman, R. F. Gariepy. Fast/Slow diffusion and collapsing sandpiles, J.Diff.Equat., 137(1997), 166-209

[EGan] L. C. Evans, W. Gangbo. Differential equations methods for the MongeKantorovich mass transfer problem, Preprint.

[EGar92] L. C. Evans, R. F. Gariepy. Measure theory and fine properties of functions, CRC Press, 1992.

[EH87] W. D. Evans, D. J. Harris. Sobolev embeddings for generalized ridged domains, Proc.London Math.Soc., 54(1987), 141-175.

[Fed59] H. Federer. Curvature measures, Trans. Am. Math. Soc., 93(1959), 418-491.

[Fed69] H. Federer. Geometric measure theory, Springer, 1969.

[Fel] M. Feldman. Convex viscosity solutions of nonlocal geometric motion of planar curves, in preparation.

[GT83] D. Gilbarg, N.Trudinger. Elliptic Partial Differential Equations of second order (2nd Ed.), Springer, 1983.

[Jan93] U.Janfalk. On certain problems concerning the p-Laplace operator, Linkoping Studies in Science and Technology, Dissertation \#326, Linkoping University, Sweden, 1993.

[Kry87] N. Krylov. Nonlinear elliptic and parabolic equations of second order, Reidel, Dordrecht, 1987.

[Son93] H. M. Soner. Motion of a set by a curvature of its boundary, J.Diff.Equat., 101(1993), 313-372.

Mathematical Sciences Research Institute, 1000 Centennial Dr., Berkeley CA 94720

E-mail address: mikhail@msri.org 